\title{
Heat shock protein 90 is involved in the regulation of HMGA2- driven growth and epithelial-to-mesenchymal transition of colorectal cancer cells
}

Chun-Yu Kao, Pei-Ming Yang, Ming-Heng Wu, Chi-Chen Huang, Yi-Chao Lee, Kuen-Haur Lee

High mobility group AT-hook 2 (HMGA2) is a nonhistone chromatin-binding protein and act as a transcriptional regulating factor involved in gene transcription. In particular, overexpression of HMGA2 has been demonstrated to associate with neoplastic transformation and tumor progression in colorectal cancer (CRC). Thus, HMGA2 is a potential therapeutic target in cancer therapy. Heat shock protein 90 (Hsp90) is a chaperone protein required for the stability and function for a number of proteins that promote the growth, mobility, and survival of cancer cells. Moreover, it has shown strong positive connections were observed between Hsp90 inhibitors and CRC, which indicated their potential for use in CRC treatment by using combination of data mining and experimental designs. However, little is known about the effect of Hsp90 inhibition on HMGA2 protein expression in CRC. In this study, we tested the hypothesis that Hsp90 may regulate HMGA2 expression and investigated the relationship between Hsp90 and HMGA2 signaling. The use of the second-generation Hsp90 inhibitor, NVP-AUY922, considerably knocked down HMGA2 expression, and the effects of Hsp90 and HMGA2 knockdown were similar. In addition, Hsp90 knockdown abrogates colocalization of Hsp90 and HMGA2 in CRC cells. Moreover, the suppression of HMGA2 protein expression in response to NVPAUY922 treatment resulted in ubiquitination and subsequent proteasome-dependant degradation of HMGA2. Furthermore, RNAi-mediated silencing of HMGA2 reduced the survival of CRC cells and increased the sensitivity of these cells to chemotherapy. Finally, we found that the NVP-AUY922-dependent mitigation of HMGA2 signaling occurred also through indirect reactivation of the tumor suppressor microRNA (miRNA), let-7a, or the inhibition of ERK-regulated HMGA2 involved in regulating the growth of CRC cells. Collectively, our studies identify the crucial role for the Hsp90-HMGA2 interaction in maintaining CRC cell survival and migration. These findings have significant implications for inhibition HMGA2-dependent tumorigenesis by clinically available Hsp90 inhibitors. 
1 Title: Heat shock protein 90 is involved in the regulation of HMGA2-driven growth and 2 epithelial-to-mesenchymal transition of colorectal cancer cells

3

4 Authors: Chun-Yu Kao ${ }^{1}$, Pei-Ming Yang ${ }^{2,5}$, Ming-Heng $\mathrm{Wu}^{3,5}$, Chi-Chen Huang ${ }^{4,5}$, Yi-Chao

5 Lee $^{4 *}$, Kuen-Haur Lee ${ }^{2 *}$

6

7 Affiliations:

$8{ }^{1}$ Department of Pediatric Surgery, Taipei Medical University-Shuang Ho Hospital, New Taipei

9 City, Taiwan

${ }^{2}$ Graduate Institute of Cancer Biology and Drug Discovery, College of Medical Science and Technology, Taipei Medical University, Taipei, Taiwan

${ }^{3}$ Graduate Institute of Translational Medicine, College of Medical Science and Technology, Taipei Medical University, Taipei, Taiwan

${ }^{4}$ Graduate Institute of Neural Regenerative Medicine, College of Medical Science and Technology, Taipei Medical University, Taipei, Taiwan

*Correspondence should be addressed to:

Yi-Chao Lee, Graduate Institute of Neural Regenerative Medicine, College of Medical Science and Technology, Taipei Medical University, No. 250, Wu-Hsing Street, Taipei 11031, Taiwan. Phone: (886)-2-27361661 ext. 7612.

Kuen-Haur Lee, Graduate Institute of Cancer Biology and Drug Discovery, College of Medical Science and Technology, Taipei Medical University, No. 250, Wu-Hsing Street, Taipei 11031, Taiwan. Phone: (886)-2-27361661 ext. 7627.

${ }^{5}$ These authors contributed equally to this work.

\section{ABSTRACT}

High mobility group AT-hook 2 (HMGA2) is a nonhistone chromatin-binding protein and act as a transcriptional regulating factor involved in gene transcription. In particular, overexpression of HMGA2 has been demonstrated to associate with neoplastic transformation and tumor progression in colorectal cancer (CRC). Thus, HMGA2 is a potential therapeutic target in cancer therapy. Heat shock protein 90 (Hsp90) is a chaperone protein required for the 
32 stability and function for a number of proteins that promote the growth, mobility, and survival of

33 cancer cells. Moreover, it has shown strong positive connections were observed between Hsp90

34 inhibitors and $\mathrm{CRC}$, which indicated their potential for use in CRC treatment by using 35 combination of data mining and experimental designs. However, little is known about the effect 36 of Hsp90 inhibition on HMGA2 protein expression in CRC. In this study, we tested the 37 hypothesis that Hsp90 may regulate HMGA2 expression and investigated the relationship 38 between Hsp90 and HMGA2 signaling. The use of the second-generation Hsp90 inhibitor, NVP39 AUY922, considerably knocked down HMGA2 expression, and the effects of Hsp90 and 40 HMGA2 knockdown were similar. In addition, Hsp90 knockdown abrogates colocalization of 41 Hsp90 and HMGA2 in CRC cells. Moreover, the suppression of HMGA2 protein expression in 42 response to NVP-AUY922 treatment resulted in ubiquitination and subsequent proteasome43 dependant degradation of HMGA2. Furthermore, RNAi-mediated silencing of HMGA2 reduced the survival of CRC cells and increased the sensitivity of these cells to chemotherapy. Finally, we found that the NVP-AUY922-dependent mitigation of HMGA2 signaling occurred also through indirect reactivation of the tumor suppressor microRNA (miRNA), let-7a, or the inhibition of ERK-regulated HMGA2 involved in regulating the growth of CRC cells. Collectively, our studies identify the crucial role for the Hsp90-HMGA2 interaction in maintaining CRC cell survival and migration. These findings have significant implications for inhibition HMGA2-dependent tumorigenesis by clinically available Hsp90 inhibitors. 
High mobility group AT-hook (HMGA) nonhistone chromatin-binding proteins, including HMGA1 (isoforms HMGA1a and HMGA1b) and HMGA2, are architectural nuclear factors involved in chromatin remodeling and gene transcription (Reeves \& Nissen, 1990). HMGA1 and HMGA2 have similar functions and are abundantly expressed in the early embryo, in which cells proliferate rapidly (Sgarra et al., 2004). However, HMGA2 cannot be detected in adult human tissues, in which it is probably completely silenced (Gattas et al., 1999; Rogalla et al., 1996). In particular, HMGA2 is weakly expressed only in preadipocytic proliferating cells (Anand \& Chada, 2000) and spermatocytes (Di Agostino et al., 2004). Conversely, several studies have reported that the association of HMGA2 overexpression with the transformation and metastatic progression of neoplastic cells suggests its causal role in carcinogenesis and tumor progression (Mahajan et al., 2010; Piscuoglio et al., 2012; Wang et al., 2011; Wend et al., 2013; Xu et al., 2004). Furthermore, the essential role of HMGA2 in cell proliferation and migration has been reported in various cancers (Malek et al., 2008; Sun et al., 2013; Xia et al., 2015; Yang et al., 2011). Thus, the HMGA2 protein is a promising biomarker for cancer detection as well as a potential molecular target in cancer therapy.

Heat shock protein 90 (Hsp90), one of the most abundant and highly conserved molecular chaperones, is essential for the stability and function of multimutated, chimeric, and overexpressed signaling proteins that promote the growth, mobility, and survival of cancer cells (Neckers, 2002). In addition, Hsp90 is involved in the maturation and stabilization of various oncogenic client proteins crucial for oncogenesis and malignant progression (Chiosis et al., 2006). Thus, Hsp90 is considered a valuable target for cancer therapy. Moreover, using a combination of microarray gene expression of 132 colorectal cancer (CRC) patients and Connectivity Map data mining, extremely strong positive connections were observed between Hsp90 inhibitors and CRC, which indicated their potential for use in CRC treatment (Su et al., 2015). However, the correlation and regulatory mechanism between Hsp90 and HMGA2 in CRC remain largely unclear. 


\section{MATERIALS AND METHODS}

\section{Chemicals, reagents, antibodies, and expression constructs}

96 NVP-AUY922 was purchased from Selleck Chemicals LLC (Houston, TX, USA). Crystal violet 97 and DMSO were obtained from Sigma (St. Louis, MO, USA). Small interfering RNA (siRNA) 98 targeting Hsp90 or HMGA2 mRNA, control siRNA, and the RNAiMax transfection reagent 99 were purchased from Life Technologies (Carlsbad, CA, USA). Rabbit antibodies against Hsp90, 100 CDK4, E-cadherin, vimentin, Twist, Snail, Slug, extracellular signal-regulated kinase (ERK), $101 \operatorname{Thr}(\mathrm{P}) 202 / \operatorname{Tyr}(\mathrm{P}) 204-E R K 1 / 2$, cAMP response element-binding protein (CREB), Ser(P)133102 CREB, focal adhesion kinase (FAK), Tyr(P)397-FAK, Lin28B, Tyr(P)705-Stat3, Stat3, and c103 Myc were obtained from Cell Signaling (Beverly, MA, USA). HMGA2 and GFP were obtained 104 from Santa Cruz Biotechnology (Santa Cruz, CA, USA). Mouse monoclonal antibody against $\beta$ 105 actin was purchased from MP Biomedicals (Irvine, CA, USA). AZD6244 was acquired from 106 Selleckchem (Houston, TX, USA).

\section{Cell culture}

108 CRC cell lines were provided by Prof. YW Cheng and Prof. H Lee (Graduate Institute of Cancer Biology and Drug Discovery, Taipei Medical University). Stable DLD-HMGA2-GFP expression cell line was provided by Dr. PM Yang (Graduate Institute of Cancer Biology and Drug

111 Discovery, Taipei Medical University). All CRC cell lines were cultured in RPMI-1640 and 112 supplemented with $10 \%$ fetal bovine serum (FBS) and $1 \%$ penicillin and streptomycin (P/S).

113 CRL-1459/CCD-18Co (noncancerous human colon cells) was provided by Prof. PJ Lu (Institute 114 of Clinical Medicine, National Cheng Kung University) and cultured in minimum essential 115 Eagle's medium and supplemented with $10 \%$ FBS and $1 \%$ penicillin and streptomycin $(\mathrm{P} / \mathrm{S})$.

\section{Cell viability assay}

117 Cell viability was determined through crystal violet staining, as described by (Kim et al., 1997).

118 In brief, the cells were plated in $96-$ well plates at 4000 cells $/ \mathrm{mL}$ and subjected to DMSO or 119 NVP-AUY922 treatment at the indicated concentrations. Viable cells were stained with $0.5 \%$ 120 crystal violet in $30 \%$ ethanol for $10 \mathrm{~min}$ at room temperature. Subsequently, the plates were 121 washed four times with tap water. After drying, the cells were lysed with a $0.1 \mathrm{M}$ sodium citrate 122 solution, and the dye uptake was measured at $550 \mathrm{~nm}$ using a 96-well plate reader. Cell viability 123 was calculated by comparing the relative dye intensities of the treated and untreated samples.

\section{Tissue microarray of CRC clinical specimens}


125 A colon adenocarcinoma tissue array was purchased from US Biomax (CO1505, containing 50

126 cases of CRC tissues with matched adjacent tissues as the controls). All tissue sections were

127 stained using a standard immunohistochemical (IHC) protocol. In brief, slides were

128 deparaffinized using serial xylene-ethanol treatment. Antigens were retrieved through boiling in

129 a sodium citrate buffer for $10 \mathrm{~min}$. Slides were blocked in 5\% normal goat serum for 1 hour at

130 room temperature. After blocking, the slides were incubated with a primary antibody against

131 HMGA2, followed by a biotin-conjugated secondary antibody, horseradish peroxidase polymer

132 (HRP), and a diaminobenzidine-tetrahydrochloride-dihydrate solution. The staining intensity was

133 scored as follows: 0 point, negative; 1 point, weakly positive; 2 points, moderately positive; and

1343 points, strongly positive.

\section{Quantitative reverse-transcription polymerase chain reaction}

136 Total RNA was extracted from the cell lines with or without drug treatment using a Qiagen

137 RNeasy kit and Qiashredder columns according to manufacturer instructions (Valencia, CA, 138 USA). One microgram of the total RNA was reverse transcribed to cDNA using a 139 SABiosciences Reaction Ready ${ }^{\mathrm{TM}}$ First Strand cDNA Synthesis Kit (Frederick, MD, USA).

140 Quantitative reverse-transcription polymerase chain reaction (RT-PCR) was performed in an

141 Applied Biosystems StepOne Plus ${ }^{\mathrm{TM}}$ Real-Time PCR System (Foster City, CA, USA) using an

142 automated baseline and threshold cycle detection. For detecting the expression levels of let-7a,

143 HMGA2, and GAPDH, the amplification and detection of specific products were performed

144 using the cycle profile of the Qiagen miScript SYBR green PCR starter kit (Valencia, CA, USA).

145 The relative gene expression level was calculated by comparing the cycle times for each target

146 PCR. The let-7a PCR Ct values were normalized by subtracting the U6 rRNA Ct value (internal

147 control) (RNU6-2_11 miScript Primer Assay; Qiagen catalog number: MS00033740). The

148 sequences of the primers used in this study are listed as follows: Let-7a: 5'-

149 UgagguaguagGUUGUAUAGUU-3'; HMGA2: forward 5'-TTCAGCCCAG-

150 GGACAACCT-3' and reverse 3'-TCTTGTTTTTGCTGCCTTTGG-5'; GAPDH: forward 5'151 AATCCCATCACCATCTTCCA-3' and reverse 3'-ACTCATGCAGCACCTCAGGT-5'.

\section{Transfection}

153 Cells were transfected with siRNAs for 48 hours using Lipofectamine 2000 (Life Technologies)

154 according to the manufacturers' instructions. The siRNA used in this study from Life 155 Technologies and their sequences were as follows: Hsp90 (siRNA ID: s6994): sense 5'- 
156 CUAUGGGUCGGUGGAACAAAtt-3' and antisense 5'-UUUGUUCCACGACCC-AUAGgt-3';

157 HMGA2 (siRNA ID: s224869): sense 5'-GGAGAAAAACGGCAAGAGtt-3' and antisense 5'-

158 CUCUUGGCCGUUUUUCUCCag-3'.

159 Immunofluorescence staining

160 HCT116 cells grown on glass coverslips were transfected with control siRNA or siHsp90,

161 respectively. At 48 hours post-transfection, the cells were fixed with 4\% ice-cold

162 paraformaldehyde at $4{ }^{\circ} \mathrm{C}$ for $20 \mathrm{~min}$ and then permeabilized with PBS with $0.5 \%$ Triton X-100

163 for $10 \mathrm{~min}$ at room temperature (RT), then washed, and blocked with 10\% goat serum in

164 phosphate-buffered saline (PBS) for 45 minutes at RT. Cells were then incubated overnight at

$1654^{\circ} \mathrm{C}$ with the mouse anti-Hsp90 (1:300; Abcam, MA, USA) or rabbit anti-HMGA2 (1:300; Santa

166 Cruz Biotechnology, TX, USA). After washing, the cells were incubated at RT for 1.5 hours with

167 Alexa-Fluor-546-conjugated goat anti-mouse IgG secondary antibody (1:500) (Invitrogen) or

168 Alexa-Fluor-488-conjugated goat anti-rabbit IgG secondary antibody (1:500) (Invitrogen). After

1693 washes, cells were mounted on glass slides in Mount medium containing DAPI (4, 6

170 diamidino-2-phenylindole;Polysciences) (Vector Laboratories, CA, USA). The images were

171 examined on an Olympus FV1000 confocal microscope (Olympus Corp., Japan).

172 Immunoprecipitation

173 The interaction between Hsp90 protein and HMGA2 was studied by immunoprecipitation

174 analysis of extracts prepared from HCT116 or DLD1 cell lines. Cells were lysed, incubated in IP

175 lysis buffer (10 mM Tris- $\mathrm{HCl} \mathrm{pH} \mathrm{7.5,} 150 \mathrm{mM} \mathrm{NaCl}$, protease inhibitor mixture) for $30 \mathrm{~min}$ on

$176 \mathrm{ice}$, and then sonicated ( 3 times for $10 \mathrm{sec}$ each). After centrifugation at $1400 \mathrm{~g}$ for $5 \mathrm{~min}$ at $4^{\circ} \mathrm{C}$,

177 the supernatants were collected from each sample and then pre-cleared by incubation with 50\%

178 protein $\mathrm{A} / \mathrm{G}$ agarose beads in the IP lysis buffer at $4^{\circ} \mathrm{C}$ for 1 hour with rocking. After removal of

179 the protein $\mathrm{A} / \mathrm{G}$ beads by centrifugation, protein concentration in each sample was measured and

180 aliquots containing $500 \mu \mathrm{g}$ of protein were incubated with primary antibodies overnight at $4^{\circ} \mathrm{C}$.

181 The immunoprecipitates bound to the protein A/G-Sepharose beads were washed, boiled and

182 analyzed by immunoblotting.

183 Western blotting

184 Cell lines were placed in a lysis buffer at $4{ }^{\circ} \mathrm{C}$ for 1 hour. Protein samples were electrophoresed 185 using 8\%-15\% SDS-polyacrylamide gel electrophoresis, as described by Su et al. (2015). 
187 HCT116 cell line was analyzed in the array panel of kinase phosphorylation profiles after DMSO 188 or NVP-AUY922 treatment (Human Phospho-Kinase Array, ARY003; R\&D Systems). This 189 array specifically screens for relative phosphorylation levels of 42 individual proteins involved in 190 cellular proliferation and survival. Each phospho-kinase array has duplicate signal spots for each 191 gene. After DMSO or NVP-AUY922 (10 nM) treatment, cell lysates were incubated with the 192 membrane. Thereafter, a cocktail of biotinylated detection antibodies, streptavidin-HRP, and 193 chemiluminescent detection reagents were used for detecting phosphorylated proteins. The bar 194 graphs were normalized by using blank spot. The dot density was scanned from the scanned X195 ray film, and images were analyzed and quantified using image analysis software (NIH-Image J).

196 In vitro migration assay

197 Assays were performed using Falcon ${ }^{\mathrm{TM}}$ cell culture inserts (8- $\mu \mathrm{m}$ pore size) in a 24-well plate 198 (BD Biosciences, San Jose, CA, USA) according to manufacturer instructions. In the migration 199 assay, HCT116 cells $\left(10^{4}\right.$ cells/well $)$ in $0.5 \mathrm{~mL}$ of serum-free medium were seeded onto the upper 200 chamber membranes that received different treatment. These membranes were previously 201 inserted into the 24-well plates containing 10\% FBS-supplemented medium. After 24 hours, the 202 cells were fixed with 100\% methanol and stained with 5\% Giemsa stain (Merck, Darmstadt, 203 Germany). Nonmigrated cells that remained in the upper chambers were removed by wiping the 204 top of the inserted membranes using a damp cotton swab, leaving only those cells that migrated 205 to the underside of the membranes. All experiments were performed in triplicate and 206 photographed under a phase-contrast microscope $(200 \times)$.

\section{Statistical analysis}

208 Statistical analyses were performed as recommended by an independent statistician. Unpaired

209 Student's $t$-test was used for analyses. All statistical analyses were performed using SPSS (SPSS,

210 Chicago, IL, USA), and all values are expressed as the mean \pm standard deviation. $p<0.05$ was

211 considered statistically significant.

\section{RESULTS}

\section{Elevated expression of HMGA2 mRNA and protein in CRC cell lines and tissues}

214 To determine the HMGA2 expression levels in these CRC patients, we first analyzed the gene 215 expression of HMGA2 in 132 CRC tumor samples: 67 primary CRC tissues, 65 metastatic tissues,

216 and nine normal colon controls. As expected, HMGA2 expression was significantly upregulated

217 in metastatic and primary CRC tissues compared with that in the normal colon controls (Fig. 
218 S1A). Similarly, the Hsp90 expression levels were analyzed in the same metastatic and primary 219 CRC tissues, and the mRNA expression levels of Hsp90 were similar to those of HMGA2 (Fig. 220 S1B). Next, to further understand the level of HMGA2 gene expression in human cancers, 221 various cancer cell lines were selected from the National Cancer Institute Cancer Genome 222 Anatomy Project gene expression database. CRC cell lines had relatively high levels of HMGA2 223 mRNA expression (Fig. 1A). Notably, HMGA2 was highly expressed in CRC cell lines among the 9 different types of cancers, thus validating its specificity in CRC. To determine the level of HMGA2 mRNA and protein expression in CRC cell lines, eight CRC cell lines and one noncancerous human colon cell line (CRL-1459) were chosen. Compared with CRL-1459, high expression of HMGA2 mRNAs and proteins were in all CRC cell lines except SW480 (Fig. S2 and Fig. 1B). Thus, the high levels of HMGA2 protein expression in HCT116 cells was attributed to its high mRNA expression. Therefore, HCT116 was chosen for further cell model experiments. HMGA2 protein expression was further examined in a colon adenocarcinoma tissue array (BioMax, Rockville, MD, USA). As shown in Fig. 1C, HMGA2 was upregulated in colon adenocarcinoma tissues of different tumor grades. The staining intensity of HMGA2 expression levels were defined on the basis of immunoexpression, as outlined in the IHC protocol (Fig. S3), and the colon adenocarcinoma tissues of all grades exhibited positive staining for HMGA2 (Fig. $1 \mathrm{C}$, right panel). These results indicate that HMGA2 expression was specific and elevated in CRC cells.

237 Effects of gene-specific inhibition of HMGA2 or Hsp90 and pharmaceutical 238 inhibition of Hsp90 were similar

239 Specific knockdown of HMGA2 inhibited cell proliferation, leading to an epithelial240 mesenchymal transition in human pancreatic cancer cells (Watanabe et al., 2009). To determine the effects of HMGA2 inhibition in CRC cells, cell proliferation and cell migration assays were performed; siHMGA2 knockdown significantly reduced mRNA expression level of HMGA2 (Fig. S4A) and the proliferation rate of siHMGA2-transfected HCT116 cells (Fig. 2A). The migration transwell assay was performed to determine the migratory abilities of the siHMGA2transfected HCT116 cells. As shown in Fig. 2B, the migratory abilities significantly reduced in approximately $43 \%$ of siHMGA2-transfected HCT116 cells compared with those in the control cells. To investigate whether the phenotype of gene-specific or pharmaceutical Hsp90 inhibition 
249 proliferation and cell migration assays in HCT116 cells. siHsp90 knockdown significantly 250 reduced mRNA expression level of Hsp90 about 50\% (Fig. S4B). The proliferation index in 251 siHsp90- and NVP-AUY922-treated HCT116 cells significantly reduced on Hsp90 knockdown 252 compared with that in the control cells (Figs. 2C and 2E). Moreover, cell migration significantly 253 reduced in the siHsp90- and NVP-AUY922-treated HCT116 cells. The inhibition rate was 254 approximately $85 \%$ and $65 \%$ after siHsp 90 and $40 \mathrm{nM}$ NVP-AUY922 treatments, respectively 255 (Figs. 2D and 2F, respectively). Thus, the effect of Hsp90 inhibition is similar to that of HMGA2 256 inhibition.

\section{Hsp90 regulates and interacts with HMGA2}

258 Hsp90 is critical in regulating cell growth (Cheung et al., 2010; Ko et al., 2012; Miyata, 2003; 259 Nagaraju et al., 2014), and HMGA2 has a well-documented role in this process (Di Cello et al., 260 2008; Malek et al., 2008; Sun et al., 2013; Wend et al., 2013); therefore, we examined whether 261 the interaction between the Hsp90 and HMGA2 exists. Thus, we first performed RNA 262 interference to deplete Hsp90 in the HCT116 cells and examined the effect of its depletion in the 263 intracellular localization of both Hsp90 and HMGA2 by immunofluorescence analysis. As

264 shown in Fig. 3A, Hsp90 siRNA-mediated endogenous Hsp90 knockdown significantly reduced 265 CDK4 (Hsp90 client protein) and HMGA2 expression, as well as induced Hsp70 (Hsp90 client 266 protein) expression in the siHsp90-transfected HCT116 cells. Further, immunofluorescence result revealed that Hsp90 (red color) co-localized with HMGA2 (green color) in the nucleus of control siRNA-transfected HCT116 cells (Fig. 3B, top panel: merged image show colocalization of Hsp90 and HMGA2; overlap of red and green: yellow). However, this phenomenon cannot observe in siHsp90 transfected HCT116 cells (Fig. 3B, bottom panel of merged image). These results indicated that the interaction between Hsp90 and HMGA2 exists. Next, we investigated whether the inhibition of HMGA2 through Hsp90-mediated inhibition by using Hsp90 inhibitor. As shown in Fig. 3C, HMGA2 protein expression was significantly reduced on NVP-AUY922treated HCT116 cells at both $40 \mathrm{nM}$ and $80 \mathrm{nM}$ concentrations. To evaluate a potential Hsp90HMGA2 interaction, we performed an immunoprecipitation assay to determine the effect of NVP-AUY922 on the physical interactions between Hsp90 and HMGA2. After NVP-AUY922 treatment, the HMGA2 protein was immunoprecipitated with an anti-HMGA2 antibody and analyzed through Western blotting with anti-Hsp90 or anti-HMGA2 antibodies. As shown in Fig. $3 \mathrm{D}$, a single band was detected using anti-Hsp90 antibody in immunoprecipitates or input lysate 
280 from NVP-AUY922-treated HCT116 cells. In addition, the protein interaction between Hsp90 281 and HMGA2 was not affected by treatment with NVP-AUY922 at $20 \mathrm{nM}$. The interaction 282 between Hsp90 and HMGA2 was also observed in DLD1 HMGA2-GFP cells to show that 283 Hsp90 was coimmunoprecipitated by the GFP antibody in DLD1 HMGA2-GFP cells (Fig. S5). 284 Hsp90 inhibitors cause degradation of Hsp90 client proteins via a proteasome-dependent 285 pathway (Basso et al., 2002). Therefore, we examined whether proteasomal degradation 286 mediates the loss of HMGA2 protein after treatment with NVP-AUY922. As shown in Fig. 3E, 287 decreased levels of CDK4 and HMGA2 by NVP-AUY 922 treatment at $40 \mathrm{nM}$ were recovered by 288 treatment with a proteasomal inhibitor, MG132, indicating the involvement of proteasomal 289 degradation in this loss of HMGA2 protein. Previous study have demonstrated that Hsp90 290 inhibitor-mediated proteasomal degradation of Hsp90 client proteins was preceded by their 291 ubiquitination (Grbovic et al., 2006); therefore, we then tested whether HMGA2 was 292 ubiquitinated prior to its degradation in NVP-AUY922-treated cells. Immunoprecipitation of 293 HMGA2 followed by Western blot analysis with an anti-ubiquitin antibody detected significantly 294 higher levels of ubiquitinated HMGA2 in the presence of the combination of MG132 and NVP295 AUY922, compared with either agent alone (Fig. 3F). Taken together, these data suggest that 296 downregulation of HMGA2 protein was a direct effect of Hsp90 inhibition and also indicate that 297 Hsp90 is necessary for the stability of HMGA2.

298 Inhibition of HMGA2 protein increased sensitivity of Hsp90 inhibitor

299 HMGA2 contributes to resistance against anticancer drugs in various cancer cell lines (Gyorffy et 300 al., 2006). Thus, HMGA2 silencing was hypothesized to increase the sensitivity to anticancer 301 drugs in cancer cells. To test this hypothesis, the HCT116 cell line with elevated HMGA2 302 expression was selected for transfection with HMGA2 small-interfering RNA oligomer 303 (siHMGA2) or scrambled oligomer (control siRNA). HMGA2 protein expression and cell 304 viability were subsequently examined. As shown in Fig. 4A (upper panel), HMGA2 protein 305 expression was significantly inhibited in siHMGA2-transfected HCT116 cells. To examine the 306 NVP-AUY922 drug sensitivity in siHMGA2-transfected HCT116 cells, a cell viability assay was 307 performed. NVP-AUY922 treatment significantly reduced the cell viability of siHMGA2308 transfected HCT116 cells compared with the control siRNA-transfected HCT116 cells (Fig. 4A, 309 bottom panel). The HMGA2, CDK4, and Hsp70 proteins expression were examined in control 310 siRNA or siHMGA2-transfected HCT116 cells in the presence of NVP-AUY922 (Fig. 4B). In 
311 contrast, we examined the NVP-AUY922 drug sensitivity in HMGA2-overexpressed CRC cells.

312 A stable cell line, DLD1 HMGA2-GFP, was established and characterized using an anti-GFP

313 antibody for Western blotting (Fig. 4C, upper panel). As expected, the proliferation index of the

314 stable DLD1 HMGA2-GFP cells significantly reduced on NVP-AUY922 treatment group

315 compared with the parental group (Fig. 4C, bottom panel). The effect of NVP-AUY922 in

316 inhibition of HMGA2 and CDK4 proteins expression was attenuated in HMGA2 stable clone

317 (Fig. 4D). These results are consistent with the previous observation that the HMGA2 expression

318 levels influence anticancer drug sensitivity.

319 HMGA2 as a master regulator of epithelial-mesenchymal transition (EMT) and 320 involved in NVP-AUY922-mediated suppression of EMT

321 To investigate the role of HMGA2 in regulating EMT of CRC cells, we investigated the effect of 322 siRNA-mediated knockdown of HMGA2 on the expression of EMT effectors in HCT116 cells.

323 As shown in Fig. 5A, HMGA2 knockdown inhibited EMT in HCT116 cells, which was 324 evidenced by reduced HMGA2-regulated mesenchymal markers (Twist, Snail, and Slug) (Li et 325 al., 2014; Tan et al., 2012; Thuault et al., 2008) as well as Vimentin expression in conjunction 326 with concomitant increases in the expression of the E-cadherin. In addition, Focal adhesion 327 kinase (FAK) activation is important for cancer motility. It has demonstrated that FAK is 328 regulated by HMGA2 in melanoma cells (Zhang et al., 2015). siHMGA2 treatment attenuated 329 the phosphorylation of FAK without affecting the total FAK in HCT116 cells (Fig. 5A). Pursuant 330 to these findings, we used the siRNA-mediated knockdown of HMGA2 to verify its effect in the 331 NVP-AUY922-mediated suppression of EMT in HCT116 cells. As shown in Fig. 5B, 332 knockdown HMGA2 expression can enhance the effect of NVP-AUY922-mediated suppression 333 of EMT in HCT116 cells to compare with control siRNA group. Next, the in vitro efficacy of 334 NVP-AUY922 in suppressing cancer cell mobility was illustrated by its dose-dependent 335 inhibition of the migration of HCT116 cells-transfected with control siRNA or siHMGA2 after 33624 hours of treatment in transwell assays. As shown in Fig. 5C and D, the migratory abilities 337 significantly reduced about 50\% of siHMGA2-transfected HCT116 cells compared with those in 338 the control cells and the number of migrating cells was significantly reduced in siHMGA2339 transfected HCT116 cells-treated with NVP-AUY922. Together, these findings suggest that 340 NVP-AUY922 can enhance the reduction of EMT in siHMGA2-transfected HCT116 cells. 
341 Phospho-kinase array for investigating NVP-AUY922-induced altered activity of 342 kinases that regulate growth and mobility of HCT116 cells

343 Several Hsp90 inhibitors have been identified to target Hsp90 client proteins, such as receptors, 344 kinases, and transcription factors, which are involved in oncogenesis (Porter et al., 2010; Trepel 345 et al., 2010). Extracellular signal-regulated kinase (ERK) and FAK have been demonstrated to be 346 regulated by Hsp90 and were involved in HMGA2-regulated CRC cell growth and mobility

347 (Chen et al., 2010; Li et al., 2014; Li et al., 2013; Ory et al., 2015; Zhang et al., 2015a). Our 348 aforementioned results (Fig. 3) demonstrated that Hsp90 might be the upstream regulator of 349 HMGA2. Therefore, using the human phospho-kinase array, we examined whether NVP350 AUY922 treatment in HCT116 cells altered the activity of kinases involved in regulating 351 HMGA2. As shown in Fig. 6A, the phosphorylation levels of ERK, FAK, and CREB were 352 significantly inhibited in NVP-AUY922-treated HCT116 cells. The CREB/HMGA2 pathway is 353 crucial in malignant transformation (Shibanuma et al., 2012). Furthermore, CREB is a 354 transcription factor and a downstream target of the ERK pathway (Qi et al., 2008). Accordingly, 355 we hypothesized that HMGA2-regulated cell growth can be inhibited using NVP-AUY922 356 treatment through NVP-AUY922-regulated ERK-CREB-HMGA2 signaling. Thus, HCT116 357 cells were dose-dependently treated with NVP-AUY922 for 48 hours, and the phosphorylation 358 status and total protein expression levels of ERK and CREB were examined. As shown in Fig. $3596 \mathrm{~B}$, Western blotting results revealed that the phosphorylation status and total protein expression 360 levels of ERK and CREB were significantly inhibited in the NVP-AUY922-treated HCT116 361 cells. To further investigate whether ERK was indeed involved in regulation of HMGA2, we 362 examined the dose effect of AZD6244, a potent ERK inhibitor, on the phosphorylation status of 363 ERK, CREB and HMGA2 proteins expression of HCT116 cells. As shown in Fig. 6C, Western 364 blotting indicated that phosphorylation status of ERK and CREB was significantly inhibited in 365 AZD6244-treated HCT116 cells at both $75 \mathrm{nM}$ and $100 \mathrm{nM}$ in conjunction with concomitant the 366 decrease expression of HMGA2. Downregulated FAK expression results in the loss of 367 mesenchymal markers and increased expression of the epithelial marker, E-cadherin, in breast 368 tumor models (Kong et al., 2012). In addition, Hsp90 inhibition disrupts FAK signaling and 369 inhibits tumor progression (Schwock et al., 2009). To understand whether FAK was involved in 370 Hsp90-regulated EMT signaling, the phosphorylation status of FAK and EMT effectors were 371 examined. NVP-AUY922 dose-dependently reduced the phosphorylation level of FAK, 
372 accompanied by parallel changes in the expression of various EMT effectors, including E-

373 cadherin, Vimentin, Twist, Snail, and Slug in HCT116 cells, with the reversal from a

374 mesenchymal to an epithelial phenotype (Fig. 6D). This result is consistent with the findings of

375 Fig. 5A to indicate Hsp90-regulated EMT signaling through HMGA2-regulated signaling.

376 Collectively, these results clearly indicate that Hsp90 can indirectly regulated HMGA2 via

377 activation of the ERK signaling pathway, and this regulatory mechanism can be inhibited by

378 treatment the Hsp90 inhibitor.

379 DISCUSSION

380 HMGA2 overexpression in various human neoplasias is associated with highly malignant 381 phenotypes, such as chemoresistance, metastasis, and poor survival (Di Cello et al., 2008;

382 Mahajan et al., 2010; Wang et al., 2011; Yang et al., 2011). HMGA2 or HMGA2-regulated

383 signaling is the preferred therapeutic target in CRC. This is the first study to recognize HMGA2

384 as a newly identified Hsp90 client protein and to propose pharmacological Hsp90 inhibition as a

385 promising strategy for impairing HMGA2 function. We demonstrated that the Hsp90 mRNA

386 expression levels in primary and metastatic CRC tissues were similar to those of HMGA2,

387 analyzed from the Gene Expression Omnibus repository (GSE21815), and reported that the

388 Hsp90 inhibitor follows a rational therapeutic approach in inhibiting HMGA2-triggered

389 tumorigenesis. The knockdown of Hsp90 using Hsp90 siRNA significantly reduced HMGA2

390 expression, and the effects of Hsp90 and HMGA2 knockdown were similar. The relationship of

391 HMGA2 and Hsp90 was examined by immunofluorescence and in vitro ubiquitination assays in

392 CRC cells. Moreover, our cell viability data clearly demonstrated that HMGA2 expression levels

393 influenced NVP-AUY922-induced drug sensitivity of the CRC cells. NVP-AUY922 treatment in

394 CRC cells significantly downregulated the regulatory activities of kinases involved in regulation

395 of HMGA2. Collectively, this is the first study to report that Hsp90 inhibitor significantly

396 suppressed HMGA2 protein expression and HMGA2-mediated regulation of cell growth and 397 mobility.

398 MiRNAs are critical in the regulation of HMGA2 protein expression (D'Angelo et al., 2015).

399 Let-7a is one of the most critical tumor suppressor miRNA that regulates HMGA2 expression

400 (Wang et al., 2013; Wu et al., 2015; Yang et al., 2014). In particular, let-7a dysregulation was

401 observed in CRC (Pallante et al., 2015). In the present study, let-7a expression was significantly

402 induced using NVP-AUY922 (40 nM) treatment in HCT116 cells (Fig. S6A), and HMGA2 
403 protein expression was simultaneously inhibited (comparison of Figs. 3C and Fig. S6A). It has 404 shown that the biogenesis of let-7a was blocked by overexpression of c-Myc/Lin28B axis in 405 cancer cells (Pang et al., 2014). In addition, it has been demonstrated that Stat3-coordinated 406 Lin28B-let-7-HMGA2 signaling to circuit initiate and maintain oncostatin M-driven EMT (Guo 407 et al., 2013). To determine whether reactivation of let-7a by treatment with Hsp90 inhibitor 408 through inhibition of c-Myc/Lin28B axis or Stat3 signaling, these proteins were detected in 409 NVP-AUY922-treated HCT116 cells. As shown in Fig. S6B, the phosphorylation status of Stat3 410 and protein expression of Lin28B and c-myc were completely inhibited on NVP-AUY922411 treated HCT116 cells at $40 \mathrm{nM}$ for 24 hours. In clinical CRC specimens, quantitative RT-PCR 412 and IHC analysis revealed downregulated let-7a expression levels and upregulated HMGA2 413 protein expression levels, respectively (unpublished data). These results show that let-7a acts as a 414 suppressor of CRC tumorigenesis, and NVP-AUY92-induced let-7a reactivation can inhibit 415 HMGA2-triggered cell growth and mobility of CRC cells.

416 HMGA2 is an architectural transcription factor and belongs to the high motility group A 417 family. This family of proteins can modify the structure of its binding partners to generate a 418 conformation that facilitates various DNA-dependent activities and influences various biological 419 processes, including cell growth, metastasis, and survival (Califano et al., 2014; Morishita et al., 420 2013). HMGA2 protein regulates the transcription of several EMT-related genes and thus is 421 closely associated with tumor invasion and metastasis (Morishita et al., 2013). HMGA2 422 upregulated the expression of Snail and Twist and downregulated the expression of E-cadherin in 423 normal murine mammary gland epithelial cells (Thuault et al., 2006). In addition, HMGA2 424 positively regulated Slug expression by directly binding to the regulatory region of the Slug 425 promoter ( $\mathrm{Li}$ et al., 2014). HMGA2 was involved in cordycepin-mediated suppression of late426 stage melanoma metastasis through the modulation of the activation of FAK and expression of 427 EMT effectors (Zhang et al., 2015b). Furthermore, FAK expression downregulation results in the 428 loss of mesenchymal markers and increased epithelial marker expression in breast tumor models 429 (Kong et al., 2012). These results reveal the criticality of HMGA2 in cancer progression, and 430 thus HMGA2 is a potential molecular target for preventing cancer progression. However, the 431 molecular mechanism of the Hsp90 inhibitor in the inhibition of metastasis remains unclear. An 432 Hsp90 inhibitor, 17-allylamino-17-demethoxygeldanamycin, inhibited prostate cancer metastasis 433 through Slug inhibition (Ding et al., 2013). This is the first study to examine the potency of a 
434 second generation Hsp90 inhibitor, NVP-AUY922, on the inhibition of migration in CRC cells 435 through the simultaneous inhibition of EMT effectors regulated by HMGA2.

436 In summary, NVP-AUY922 reduced the activity and expression of ERK and CREB and 437 suppressed CRC cell growth. In addition, NVP-AUY922 downregulated the expression of 438 HMGA2 and HMGA2-mediated EMT effectors, which suppressed cell motility, suggesting that 439 NVP-AUY922 not only regulates the growth of CRC cells but also its dissemination.

\section{CONCLUSIONS}

441 Our study is the first to identify the interaction between Hsp90 and HMGA2 and that the Hsp90 442 inhibitor has therapeutic potential to inhibit HMGA2-triggered tumorigenesis. Moreover, our 443 findings clarify the downregulation of HMGA2 was a direct effect of Hsp90 inhibition and also 444 indicate that Hsp90 is necessary for the stability of HMGA2. Moreover, Hsp90 inhibitor also can 445 indirectly regulated HMGA2 via inactivation of the ERK signaling pathway or reactivation of 446 let-7a.

\section{REFERENCES}

448

449

450

451

452

453

454

455

456

457

458

459

460

461

462

463

464

465

466

467

468

469

470

471

472

Anand A, and Chada K. 2000. In vivo modulation of Hmgic reduces obesity. Nat Genet 24:377380 DOI $10.1038 / 74207$.

Basso AD, Solit DB, Chiosis G, Giri B, Tsichlis P, and Rosen N. 2002. Akt forms an intracellular complex with heat shock protein 90 (Hsp90) and Cdc37 and is destabilized by inhibitors of Hsp90 function. $J$ Biol Chem 277:39858-39866 DOI 10.1074/jbc.M206322200.

Califano D, Pignata S, Losito NS, Ottaiano A, Greggi S, De Simone V, Cecere S, Aiello C, Esposito F, Fusco A, and Chiappetta G. 2014. High HMGA2 expression and high body mass index negatively affect the prognosis of patients with ovarian cancer. J Cell Physiol 229:53-59 DOI 10.1002/jcp.24416.

Chen JS, Hsu YM, Chen CC, Chen LL, Lee CC, Huang TS. 2010. Secreted heat shock protein 90alpha induces colorectal cancer cell invasion through CD91/LRP-1 and NF-kappaBmediated integrin alphaV expression. $J$ Biol Chem 285: 25458-66. DOI 10.1074/jbc.M110.139345.

Cheung CH, Chen HH, Cheng LT, Lyu KW, Kanwar JR, and Chang JY. 2010. Targeting Hsp90 with small molecule inhibitors induces the over-expression of the anti-apoptotic molecule, survivin, in human A549, HONE-1 and HT-29 cancer cells. Mol Cancer 9:77 DOI 10.1186/1476-4598-9-77.

Chiosis G, Caldas Lopes E, and Solit D. 2006. Heat shock protein-90 inhibitors: a chronicle from geldanamycin to today's agents. Curr Opin Investig Drugs 7:534-541.

D'Angelo D, Esposito F, and Fusco A. 2015. Epigenetic Mechanisms Leading to Overexpression of HMGA Proteins in Human Pituitary Adenomas. Front Med (Lausanne) 2:39 DOI 10.3389/fmed.2015.00039.

Di Agostino S, Fedele M, Chieffi P, Fusco A, Rossi P, Geremia R, and Sette C. 2004. Phosphorylation of high-mobility group protein A2 by Nek2 kinase during the first 
meiotic division in mouse spermatocytes. Mol Biol Cell 15:1224-1232 DOI 10.1091/mbc.E03-09-0638.

Di Cello F, Hillion J, Hristov A, Wood LJ, Mukherjee M, Schuldenfrei A, Kowalski J, Bhattacharya R, Ashfaq R, and Resar LM. 2008. HMGA2 participates in transformation in human lung cancer. Mol Cancer Res 6:743-750 DOI 10.1158/1541-7786.MCR-070095.

Ding G, Feng C, Jiang H, Ding Q, Zhang L, Na R, Xu H, and Liu J. 2013. Combination of rapamycin, CI-1040, and 17-AAG inhibits metastatic capacity of prostate cancer via Slug inhibition. PLoS One 8:e77400 DOI 10.1371/journal.pone.0077400.

Gattas GJ, Quade BJ, Nowak RA, and Morton CC. 1999. HMGIC expression in human adult and fetal tissues and in uterine leiomyomata. Genes Chromosomes Cancer 25:316-322 DOI 10.1002/(SICI)1098-2264(199908)25:4

Grbovic OM, Basso AD, Sawai A, Ye Q, Friedlander P, Solit D, and Rosen N. 2006. V600E BRaf requires the Hsp90 chaperone for stability and is degraded in response to Hsp90 inhibitors. Proc Natl Acad Sci U S A 103:57-62 DOI 10.1073/pnas.0609973103.

Guo L, Chen C, Shi M, Wang F, Chen X, Diao D, Hu M, Yu M, Qian L, Guo N. 2013. Stat3coordinated Lin-28-let-7-HMGA2 and miR-200-ZEB1 circuits initiate and maintain oncostatin M-driven epithelial-mesenchymal transition. Oncogene 32:5272-82. DOI 10.1038/onc.2012.573.

Gyorffy B, Surowiak P, Kiesslich O, Denkert C, Schafer R, Dietel M, and Lage H. 2006. Gene expression profiling of 30 cancer cell lines predicts resistance towards 11 anticancer drugs at clinically achieved concentrations. Int $J$ Cancer 118:1699-1712 DOI 10.1002/ijc.21570.

Kim YM, Talanian RV, and Billiar TR. 1997. Nitric oxide inhibits apoptosis by preventing increases in caspase-3-like activity via two distinct mechanisms. J Biol Chem 272:3113831148 DOI 10.1074/jbc.272.49.31138.

Ko JC, Chen HJ, Huang YC, Tseng SC, Weng SH, Wo TY, Huang YJ, Chiu HC, Tsai MS, Chiou RY, and Lin YW. 2012. HSP90 inhibition induces cytotoxicity via downregulation of Rad51 expression and DNA repair capacity in non-small cell lung cancer cells. Regul Toxicol Pharmacol 64:415-424 DOI 10.1016/j.yrtph.2012.10.003.

Kong X, Li G, Yuan Y, He Y, Wu X, Zhang W, Wu Z, Chen T, Wu W, Lobie PE, and Zhu T. 2012. MicroRNA-7 inhibits epithelial-to-mesenchymal transition and metastasis of breast cancer cells via targeting FAK expression. PLoS One 7:e41523 DOI 10.1371/journal.pone.0041523.

Li Y, Zhao Z, Xu C, Zhou Z, Zhu Z, and You T. 2014. HMGA2 induces transcription factor Slug expression to promote epithelial-to-mesenchymal transition and contributes to colon cancer progression. Cancer Lett 355:130-140 DOI 10.1016/j.canlet.2014.09.007.

Li Z, Zhang Y, Ramanujan K, Ma Y, Kirsch DG, and Glass DJ. 2013. Oncogenic NRAS, required for pathogenesis of embryonic rhabdomyosarcoma, relies upon the HMGA2IGF2BP2 pathway. Cancer Res 73:3041-3050 DOI 10.1158/0008-5472.CAN-12-3947.

Mahajan A, Liu Z, Gellert L, Zou X, Yang G, Lee P, Yang X, and Wei JJ. 2010. HMGA2: a biomarker significantly overexpressed in high-grade ovarian serous carcinoma. Mod Pathol 23:673-681 DOI 10.1038/modpathol.2010.49.

Malek A, Bakhidze E, Noske A, Sers C, Aigner A, Schafer R, and Tchernitsa O. 2008. HMGA2 gene is a promising target for ovarian cancer silencing therapy. Int $J$ Cancer 123:348-356 DOI 10.1002/ijc.23491. 
519

520

521

522

523

524

525

526

527

528

529

530

531

532

533

534

535

536

537

538

539

540

541

542

543

544

545

546

547

548

549

550

551

552

553

554

555

556

557

558

559

560

561

562

563

564

Miyata Y. 2003. [Molecular chaperone HSP90 as a novel target for cancer chemotherapy]. Nihon Yakurigaku Zasshi 121:33-42.

Morishita A, Zaidi MR, Mitoro A, Sankarasharma D, Szabolcs M, Okada Y, D'Armiento J, and Chada K. 2013. HMGA2 is a driver of tumor metastasis. Cancer Res 73:4289-4299 DOI 10.1158/0008-5472.CAN-12-3848.

Nagaraju GP, Alese OB, Landry J, Diaz R, and El-Rayes BF. 2014. HSP90 inhibition downregulates thymidylate synthase and sensitizes colorectal cancer cell lines to the effect of 5FU-based chemotherapy. Oncotarget 5:9980-9991 DOI 10.18632/oncotarget.2484.

Neckers L. 2002. Hsp90 inhibitors as novel cancer chemotherapeutic agents. Trends Mol Med 8:S55-61 DOI 10.1016/S1471-4914(02)02316-X.

Ory B, Baud'huin M, Verrecchia F, Brounais-Le Royer B, Quillard T, Amiaud J, Battaglia S, Heymann D, Redini F, Lamoureux F. 2015. Blocking HSP90 addiction inhibits tumor cell proliferation, metastasis development and synergistically acts with zoledronic acid to delay osteosarcoma progression. Clin Cancer Res Dec 28. DOI 10.1158/1078-0432

Pallante P, Sepe R, Puca F, and Fusco A. 2015. High mobility group a proteins as tumor markers. Front Med (Lausanne) 2:15 DOI 10.3389/fmed.2015.00015.

Pang M, Wu G, Hou X, Hou N, Liang L, Jia G, Shuai P, Luo B, Wang K, Li G. 2014. LIN28B promotes colon cancer migration and recurrence. PLoS One. 9(10):e109169. DOI 10.1371/journal.pone.0109169.

Piscuoglio S, Zlobec I, Pallante P, Sepe R, Esposito F, Zimmermann A, Diamantis I, Terracciano L, Fusco A, and Karamitopoulou E. 2012. HMGA1 and HMGA2 protein expression correlates with advanced tumour grade and lymph node metastasis in pancreatic adenocarcinoma. Histopathology 60:397-404 DOI 10.1111/j.1365-2559.2011.04121.x.

Porter JR, Fritz CC, and Depew KM. 2010. Discovery and development of Hsp90 inhibitors: a promising pathway for cancer therapy. Curr Opin Chem Biol 14:412-420 DOI 10.1016/j.cbpa.2010.03.019.

Qi X, Lin W, Li J, Li H, Wang W, Wang D, and Sun M. 2008. Fluoxetine increases the activity of the ERK-CREB signal system and alleviates the depressive-like behavior in rats exposed to chronic forced swim stress. Neurobiol Dis 31:278-285 DOI 10.1016/j.nbd.2008.05.003.

Reeves R, and Nissen MS. 1990. The A.T-DNA-binding domain of mammalian high mobility group I chromosomal proteins. A novel peptide motif for recognizing DNA structure. $J$ Biol Chem 265:8573-8582

Rogalla P, Drechsler K, Frey G, Hennig Y, Helmke B, Bonk U, and Bullerdiek J. 1996. HMGI-C expression patterns in human tissues. Implications for the genesis of frequent mesenchymal tumors. Am J Pathol 149:775-779.

Schwock J, Dhani N, Cao MP, Zheng J, Clarkson R, Radulovich N, Navab R, Horn LC, and Hedley DW. 2009. Targeting focal adhesion kinase with dominant-negative FRNK or Hsp90 inhibitor 17-DMAG suppresses tumor growth and metastasis of SiHa cervical xenografts. Cancer Res 69:4750-4759 DOI 10.1158/0008-5472.CAN-09-0454.

Sgarra R, Rustighi A, Tessari MA, Di Bernardo J, Altamura S, Fusco A, Manfioletti G, and Giancotti V. 2004. Nuclear phosphoproteins HMGA and their relationship with chromatin structure and cancer. FEBS Lett 574:1-8 DOI 10.1016/j.febslet.2004.08.013.

Shibanuma M, Ishikawa F, Kobayashi M, Katayama K, Miyoshi H, Wakamatsu M, Mori K, and Nose K. 2012. Critical roles of the cAMP-responsive element-binding protein-mediated 
565

566

567

568

569

570

571

572

573

574

575

576

577

578

579

580

581

582

583

584

585

586

587

588

589

590

591

592

593

594

595

596

597

598

599

600

601

602

603

604

605

606

607

608

609

610 pathway in disorganized epithelial phenotypes caused by mitochondrial dysfunction. Cancer Sci 103:1803-1810 DOI 10.1111/j.1349-7006.2012.02369.x.

Su YH, Tang WC, Cheng YW, Sia P, Huang CC, Lee YC, Jiang HY, Wu MH, Lai IL, Lee JW, and Lee KH. 2015. Targeting of multiple oncogenic signaling pathways by Hsp90 inhibitor alone or in combination with berberine for treatment of colorectal cancer. Biochim Biophys Acta DOI 10.1016/j.bbamcr.2015.05.012.

Sun M, Song CX, Huang H, Frankenberger CA, Sankarasharma D, Gomes S, Chen P, Chen J, Chada KK, He C, and Rosner MR. 2013. HMGA2/TET1/HOXA9 signaling pathway regulates breast cancer growth and metastasis. Proc Natl Acad Sci U S A 110:9920-9925 DOI 10.1073/pnas.1305172110.

Tan EJ, Thuault S, Caja L, Carletti T, Heldin CH, and Moustakas A. 2012. Regulation of transcription factor Twist expression by the DNA architectural protein high mobility group A2 during epithelial-to-mesenchymal transition. J Biol Chem 287:7134-7145 DOI 10.1074/jbc.M111.291385.

Thuault S, Tan EJ, Peinado H, Cano A, Heldin CH, and Moustakas A. 2008. HMGA2 and Smads co-regulate SNAIL1 expression during induction of epithelial-to-mesenchymal transition. J Biol Chem 283:33437-33446 DOI 10.1074/jbc.M802016200.

Thuault S, Valcourt U, Petersen M, Manfioletti G, Heldin CH, and Moustakas A. 2006. Transforming growth factor-beta employs HMGA2 to elicit epithelial-mesenchymal transition. J Cell Biol 174:175-183 DOI 10.1083/jcb.200512110.

Trepel J, Mollapour M, Giaccone G, and Neckers L. 2010. Targeting the dynamic HSP90 complex in cancer. Nat Rev Cancer 10:537-549 DOI 10.1038/nrc2887.

Wang X, Liu X, Li AY, Chen L, Lai L, Lin HH, Hu S, Yao L, Peng J, Loera S, Xue L, Zhou B, Zhou L, Zheng S, Chu P, Zhang S, Ann DK, and Yen Y. 2011. Overexpression of HMGA2 promotes metastasis and impacts survival of colorectal cancers. Clin Cancer Res 17:2570-2580 DOI 10.1158/1078-0432.CCR-10-2542.

Wang YY, Ren T, Cai YY, and He XY. 2013. MicroRNA let-7a inhibits the proliferation and invasion of nonsmall cell lung cancer cell line 95D by regulating K-Ras and HMGA2 gene expression. Cancer Biother Radiopharm 28:131-137 DOI 10.1089/cbr.2012.1307.

Watanabe S, Ueda Y, Akaboshi S, Hino Y, Sekita Y, and Nakao M. 2009. HMGA2 maintains oncogenic RAS-induced epithelial-mesenchymal transition in human pancreatic cancer cells. Am J Pathol 174:854-868 DOI 10.2353/ajpath.2009.080523.

Wend P, Runke S, Wend K, Anchondo B, Yesayan M, Jardon M, Hardie N, Loddenkemper C, Ulasov I, Lesniak MS, Wolsky R, Bentolila LA, Grant SG, Elashoff D, Lehr S, Latimer JJ, Bose S, Sattar H, Krum SA, and Miranda-Carboni GA. 2013. WNT10B/beta-catenin signalling induces HMGA2 and proliferation in metastatic triple-negative breast cancer. EMBO Mol Med 5:264-279 DOI 10.1002/emmm.201201320.

Wu A, Wu K, Li J, Mo Y, Lin Y, Wang Y, Shen X, Li S, Li L, and Yang Z. 2015. Let-7a inhibits migration, invasion and epithelial-mesenchymal transition by targeting HMGA2 in nasopharyngeal carcinoma. J Transl Med 13:105 DOI 10.1186/s12967-015-0462-8.

Xia YY, Yin L, Jiang N, Guo WJ, Tian H, Jiang XS, Wu J, Chen M, Wu JZ, and He X. 2015. Downregulating HMGA2 attenuates epithelial-mesenchymal transition-induced invasion and migration in nasopharyngeal cancer cells. Biochem Biophys Res Commun 463:357363 DOI 10.1016/j.bbrc.2015.05.068.

Xu Y, Sumter TF, Bhattacharya R, Tesfaye A, Fuchs EJ, Wood LJ, Huso DL, and Resar LM. 2004. The HMG-I oncogene causes highly penetrant, aggressive lymphoid malignancy in

PeerJ reviewing PDF | (2015:10:7030:2:0:NEW 20 Jan 2016) 
611

612

613

614

615

616

617

618

619

620

621

622

623

624

625

626

627

628

629

630

\section{Figure legends}

632 Figure 1 HMGA2 was overexpressed in colorectal cancer (CRC) cell lines and tumors. (A)

transgenic mice and is overexpressed in human leukemia. Cancer Res 64:3371-3375 DOI 10.1158/0008-5472.CAN-04-0044.

Yang GL, Zhang LH, Bo JJ, Hou KL, Cai X, Chen YY, Li H, Liu DM, and Huang YR. 2011. Overexpression of HMGA2 in bladder cancer and its association with clinicopathologic features and prognosis HMGA2 as a prognostic marker of bladder cancer. Eur J Surg Oncol 37:265-271 DOI 10.1016/j.ejso.2011.01.004.

Yang MY, Chen MT, Huang PI, Wang CY, Chang YC, Yang YP, Lo WL, Sung WH, Liao YW, Lee YY, Chang YL, Tseng LM, Chen YW, and Ma HI. 2014. Nuclear Localization Signal-enhanced Polyurethane-Short Branch Polyethylenimine-mediated Delivery of Let7a Inhibited Cancer Stem-like Properties by Targeting the 3'UTR of HMGA2 in Anaplastic Astrocytoma. Cell Transplant DOI 10.3727/096368914X682107.

Zhang P, Bai H, Liu G, Wang H, Chen F, Zhang B, Zeng P, Wu C, Peng C, Huang C, Song Y, and Song E. 2015a. MicroRNA-33b, upregulated by EF24, a curcumin analog, suppresses the epithelial-to-mesenchymal transition (EMT) and migratory potential of melanoma cells by targeting HMGA2. Toxicol Lett 234:151-161 DOI 10.1016/j.toxlet.2015.02.018.

Zhang P, Huang C, Fu C, Tian Y, Hu Y, Wang B, Strasner A, Song Y, and Song E. 2015 b. Cordycepin (3'-deoxyadenosine) suppressed HMGA2, Twist1 and ZEB1-dependent melanoma invasion and metastasis by targeting miR-33b. Oncotarget 6:9834-9853 DOI 10.1016/j.toxlet.2015.02.018.

Gene expression levels of HMGA2 protein in various human cancer cell lines. (B) HMGA2 protein analysis was conducted on proteins isolated from eight CRC cell lines and one noncancerous human colon cell line (CRL-1459). (C) Left panel: Representative immunohistochemical (IHC) images of HMGA2 expression on tissue microarray containing paired normal tissues and tumors of three CRC patients with different tumor grades. Right panel: HMGA2 protein expression levels obtained from the IHC results. The percentage of cases is plotted on the y-axis, and the type of sample is plotted on the x-axis; the color indicates the HMGA2 expression levels.

Figure 2 Effects of gene-specific inhibition of HMGA2 or Hsp90 and pharmaceutical inhibition of Hsp90 were similar. Cell viability assay (A, C, and E) and cell migration analysis $(\mathrm{B}, \mathrm{D}$, and F) were performed to determine the viability and migratory ability of HCT116 cells treated with siHMGA2, siHSp90, and various concentrations of NVP-AUY922 for 48 hours (cell viability assay) or 24 hours (cell migration assay), respectively. ${ }^{*} p<0.05, * * p<0.01, * * * p<0.001$. All experiments were performed in three independent experiments. 
647 Figure 3 Direct interaction between HMGA2 and Hsp90. (A) CDK4, Hsp70, and HMGA2 648 were detected in siHsp90-transfected HCT116 cells. (B) Subcellular colocalization of HMGA2 649 and Hsp90. HCT116 cells were stained with DAPI (blue, nuclear stain) and antibodies to Hsp90 650 (red) or HMGA2 (green), and confocal images were acquired at 40× magnification. (C) HCT116 651 cells were treated with NVP-AUY922 at the indicated concentrations for 48 hours. Cell extracts 652 were analyzed using Western blotting with the antibodies for CDK4, Hsp70, and HMGA2, 653 respectively. (D) HCT116 cells were treated with NVP-AUY922 for 48 hours, HMGA2 was 654 immunoprecipitated from 500- $\mu$ g cell lysate, and resultant blots were probed for Hsp90 and 655 HMGA2 antibodies, respectively. (E) The proteasome inhibitor MG132 (1 $\mu \mathrm{M}, 24$ hours) 656 protected against NVP-AUY922-facilitated suppression of HMGA2 expression in HCT116 cells. 657 HCT116 cells were treated with $20 \mathrm{nM}$ or $40 \mathrm{nM}$ alone for 48 hours, or NVP-AUY922 658 pretreated for 24 hours and combination with MG132 for an additional 24 hours, and cell lysates 659 were subjected to Western blot analysis using anti-CDK4, anti-Hsp70, anti-HMGA2, and anti- $\beta$ 660 actin antibodies. (F) HCT116 cells were treated as above, and proteins extracts were 661 immunoprecipitated (IP) with anti-HMGA2. The ubiquitination of HMGA2 was analyzed by 662 Western blotting with anti-ubiquitin. All experiments were performed in three independent 663 experiments.

664 Figure 4 Expression levels of HMGA2 are responsible for NVP-AUY922 drug sensitivity.

665 (A) Upper panel: HMGA2 was detected in siHMGA2-transfected HCT116 cells. Bottom panel: 666 HCT116 cells were transfected with control siRNA and siHMGA2 (100 nM) for 48 hours and 667 subsequently incubated with NVP-AUY922 at the indicated concentrations for an additional 48 668 hours. A cell viability assay was performed to determine the viability of cells treated with 669 various NVP-AUY922 concentrations. Bars, SD $(\mathrm{n}=6)$. (B) Western blot analysis of proteins 670 expression of HMGA2, CDK4, and Hsp70 in HCT116 cells transfected with control siRNA or 671 siHMGA2 for 48 hours and subsequently incubated with NVP-AUY922 at the indicated 672 concentrations for an additional 48 hours. (C) Upper panel: Western blotting with anti-GFP 673 antibody of the parental and stable HMGA2-GFP groups of HCT116 cells. Bottom panel: Cell 674 proliferation assays of the parental and stable HMGA2-GFP groups of HCT116 cells treated with 675 NVP-AUY922 at the indicated concentrations for 48 hours. Bars, SD $(\mathrm{n}=6) . * p<0.05, * * p<0.01$, 676 $* * * p<0.001$. (D) Western blot analysis of proteins expression of HMGA2, CDK4, and Hsp70 in 
677 NVP-AUY922-treated of parental or HMGA2-GFP stable expression of DLD1 cells. All 678 experiments were performed in three independent experiments.

679 Figure 5 Knockdown HMGA2 expression can enhance the effect of NVP-AUY922680 mediated suppression of EMT and migratory ability of HCT116 cells. (A) siRNA-mediated 681 knockdown of HMGA2 inhibited HMGA2-regulated EMT in HCT116 cells, as revealed by loss 682 of mesenchymal markers Twist, Snail, Slug, Vimentin, and reduction the phosphorylation level 683 of FAK and gain of epithelial marker E-cadherin. (B) Effect of siRNA-mediated knockdown of 684 HMGA2 on NVP-AUY922-mediated reversal of mesenchymal character in HCT116 cells. (C 685 and D) Concentration-dependent effects of NVP-AUY922 on the migratory activity of HCT116 686 cells after 24 hours of treatment. $* * p<0.01, * * * p<0.001$. All experiments were performed in 687 three independent experiments.

688 Figure 6 Human phospho-kinase array analysis in response to NVP-AUY922 treatment in 689 HCT116 cells. (A) HMGA2-associated kinases, ERK, CREB, and FAK, were significantly 690 downregulated on NVP-AUY922 treatment. (B) Western blotting results of the concentration691 dependent effects of NVP-AUY922 on the phosphorylation and expression of ERK and CREB in 692 HCT116 cells. (C) Western blotting revealed the dose effect of AZD6244 on the phosphorylation 693 status of ERK and CREB as well as HMGA2 proteins expression of HCT116 cells. (D) Western 694 blotting results of the concentration-dependent effects of NVP-AUY922 on the phosphorylation 695 and expression of FAK and various EMT effectors of HCT116 cells. All experiments were 696 performed in three independent experiments.

697

698 


\section{1}

HMGA2 was overexpressed in colorectal cancer (CRC) cell lines and tumors.

(A) Gene expression levels of HMGA2 protein in various human cancer cell lines. (B) HMGA2 protein analysis was conducted on proteins isolated from eight CRC cell lines and one noncancerous human colon cell line (CRL-1459). (C) Left panel: Representative immunohistochemical $(\mathrm{IHC})$ images of HMGA2 expression on tissue microarray containing paired normal tissues and tumors of three CRC patients with different tumor grades. Right panel: HMGA2 protein expression levels obtained from the IHC results. The percentage of cases is plotted on the $y$-axis, and the type of sample is plotted on the x-axis; the color indicates the HMGA2 expression levels.

A

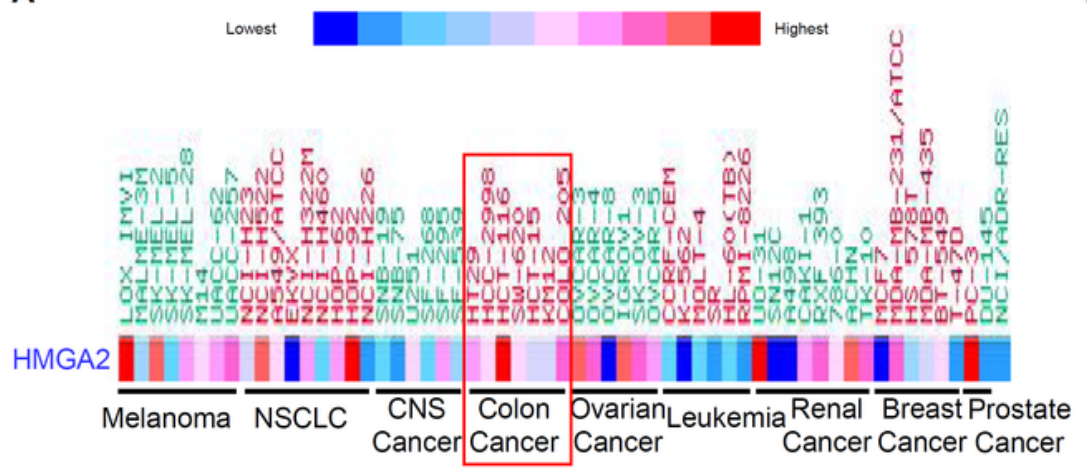

C Brown: HMGA2

Blue: Hematoxylin

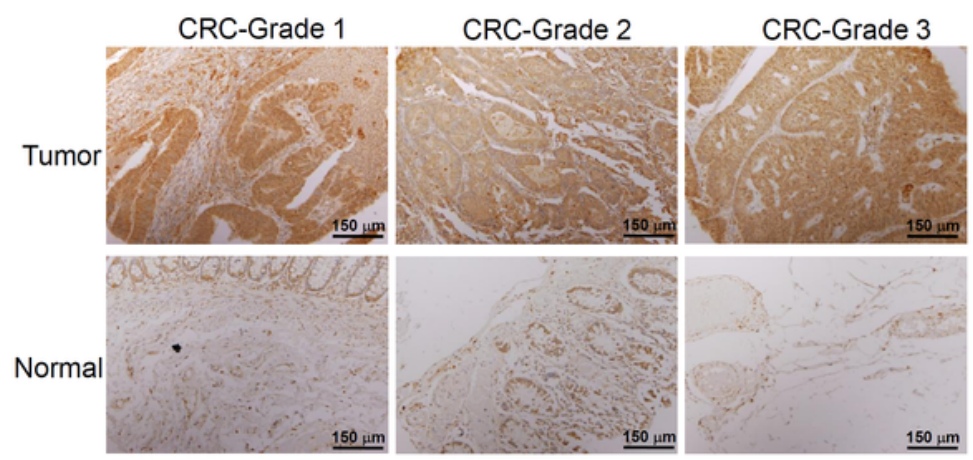

B
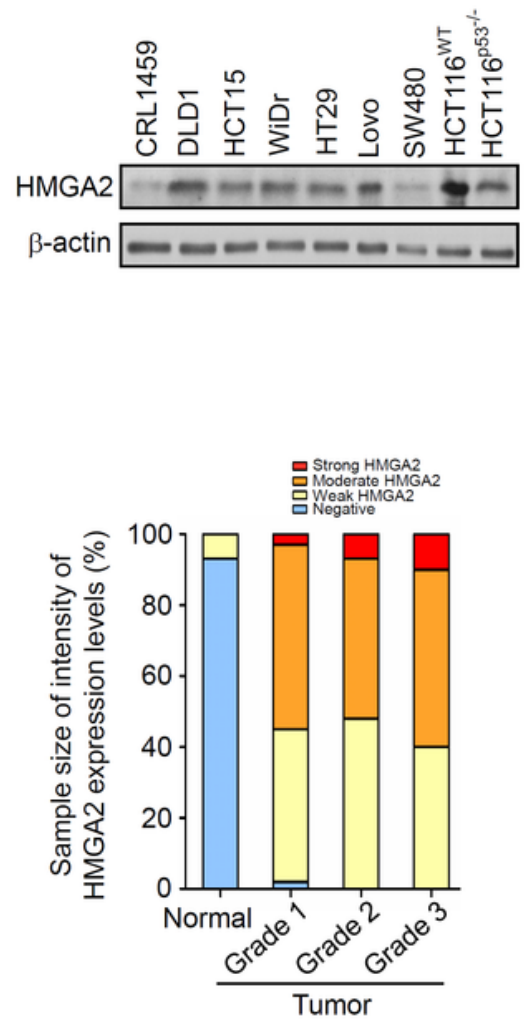

Fig.1 
2

Effects of gene-specific inhibition of HMGA2 or Hsp90 and pharmaceutical inhibition of Hsp90 were similar.

Cell viability assay $(A, C$, and $E$ ) and cell migration analysis $(B, D$, and $F)$ were performed to determine the viability and migratory ability of HCT116 cells treated with siHMGA2, siHSp90, and various concentrations of NVP-AUY922 for 48 hours (cell viability assay) or 24 hours (cell migration assay), respectively. ${ }^{*} p<0.05,{ }^{* *} p<0.01,{ }^{* * *} p<0.001$. All experiments were performed in three independent experiments.

A

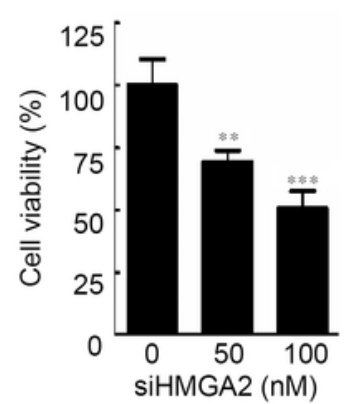

B
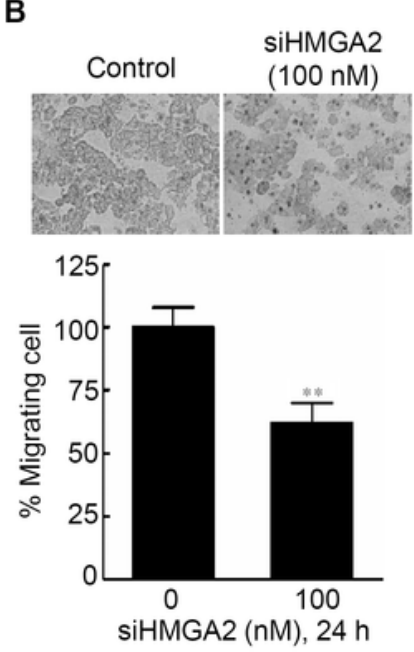

C

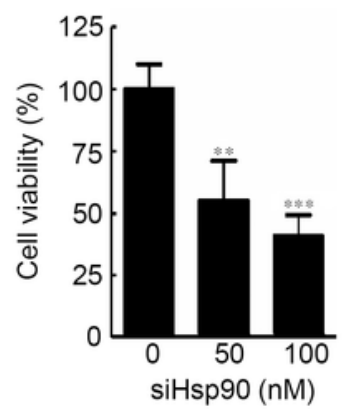

D
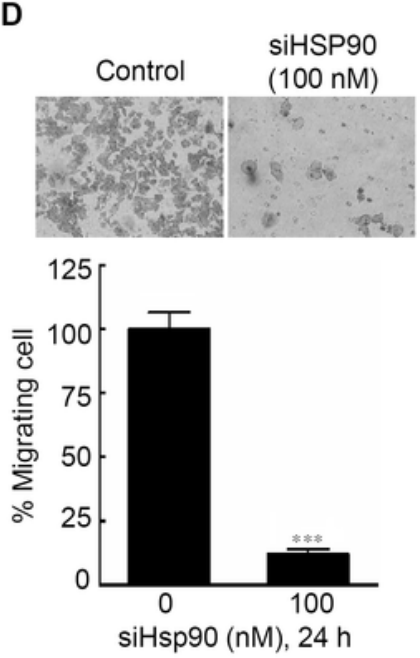

E

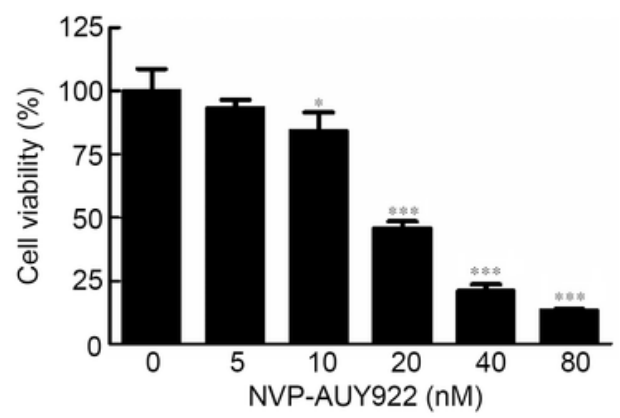

F
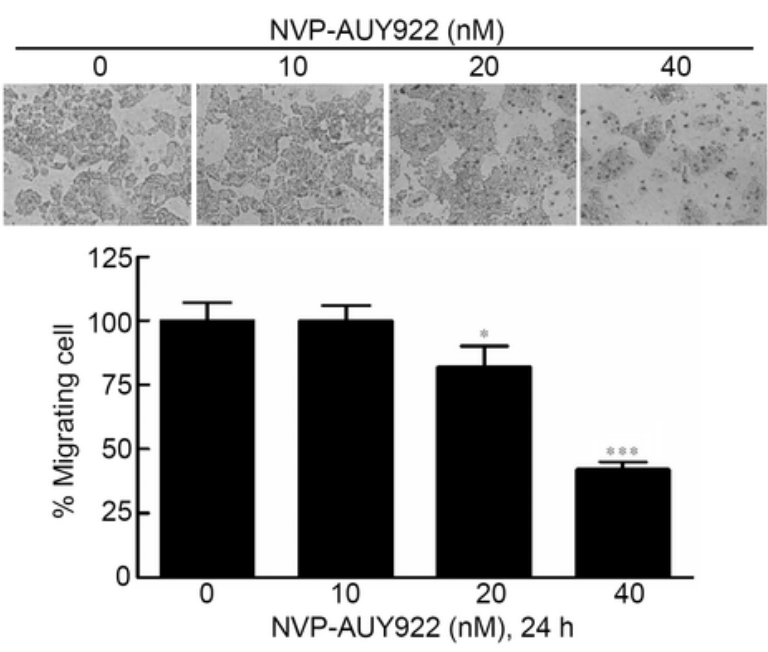

Fig.2 


\section{3}

Direct interaction between HMGA2 and Hsp90.

(A) CDK4, Hsp70, and HMGA2 were detected in siHsp90-transfected HCT116 cells. (B) Subcellular colocalization of HMGA2 and Hsp90. HCT116 cells were stained with DAPI (blue, nuclear stain) and antibodies to Hsp90 (red) or HMGA2 (green), and confocal images were acquired at 40x magnification. (C) HCT116 cells were treated with NVP-AUY922 at the indicated concentrations for 48 hours. Cell extracts were analyzed using Western blotting with the antibodies for CDK4, Hsp70, and HMGA2, respectively. (D) HCT116 cells were treated with NVP-AUY922 for 48 hours, HMGA2 was immunoprecipitated from $500-\mu \mathrm{g}$ cell lysate, and resultant blots were probed for Hsp90 and HMGA2 antibodies, respectively. (E) The proteasome inhibitor MG132 (1 $\mu \mathrm{M}, 24$ hours) protected against NVP-AUY922-facilitated suppression of HMGA2 expression in HCT116 cells. HCT116 cells were treated with $20 \mathrm{nM}$ or $40 \mathrm{nM}$ alone for 48 hours, or NVP-AUY922 pretreated for 24 hours and combination with MG132 for an additional 24 hours, and cell lysates were subjected to Western blot analysis using anti-CDK4, anti-Hsp70, anti-HMGA2, and anti- $\beta$-actin antibodies. (F) HCT116 cells were treated as above, and proteins extracts were immunoprecipitated (IP) with anti-HMGA2. The ubiquitination of HMGA2 was analyzed by Western blotting with anti-ubiquitin. All experiments were performed in three independent experiments. 
A

B
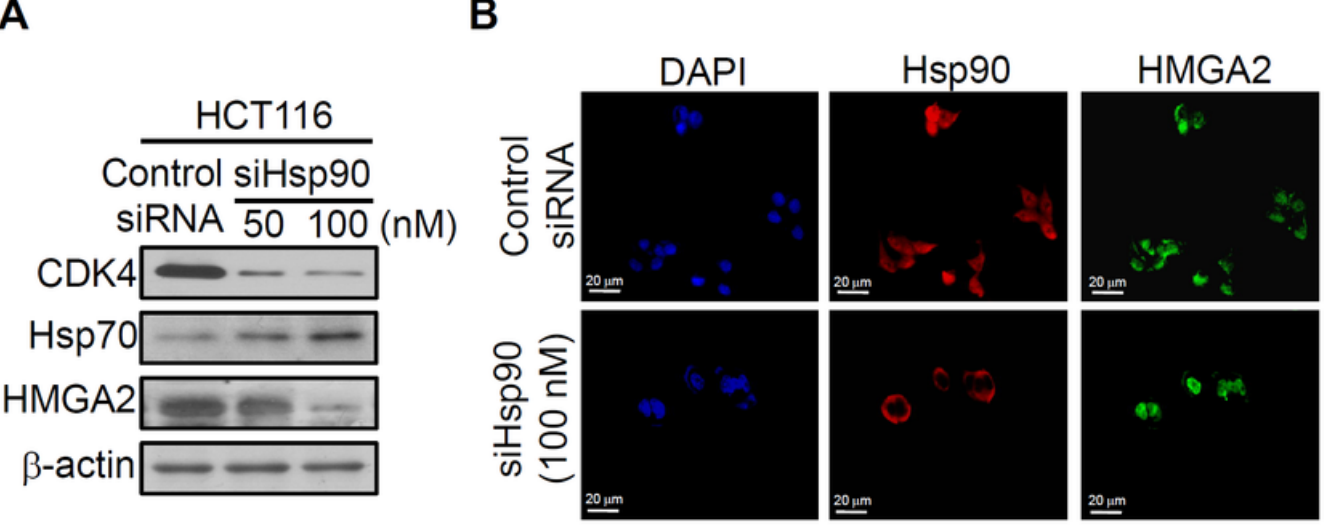

Hsp90+
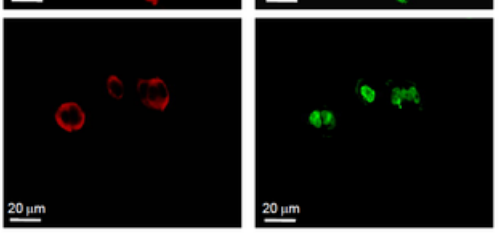

HMGA2

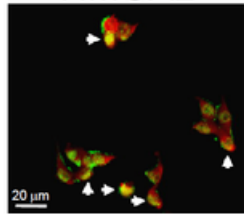

C

D
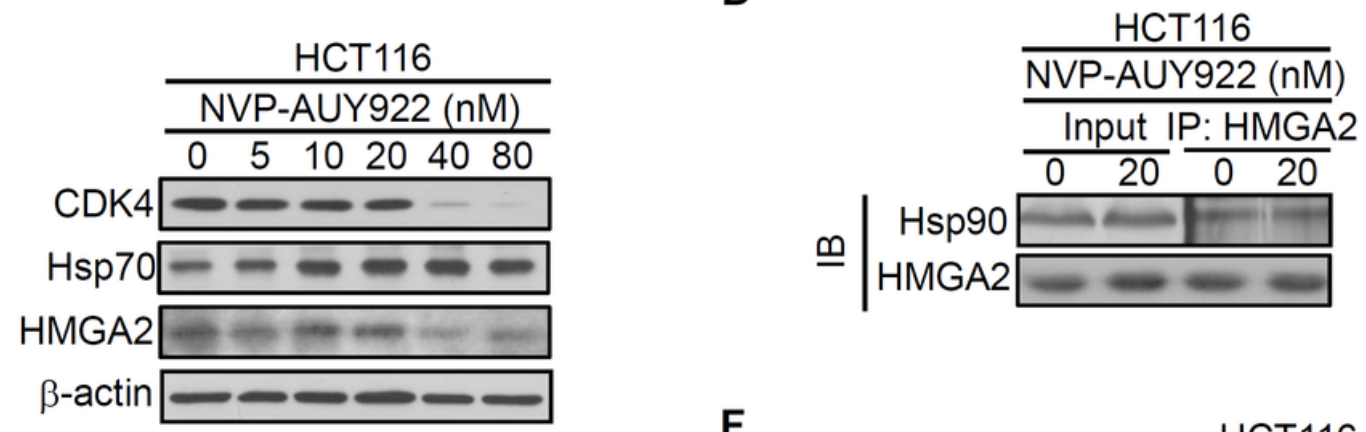

E

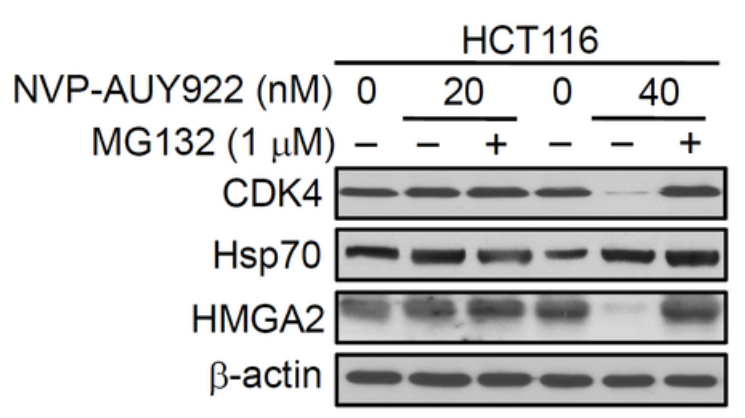

$\mathbf{F}$

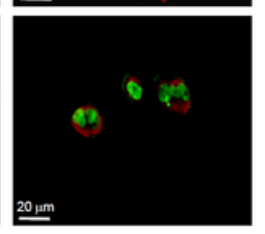

\section{E}
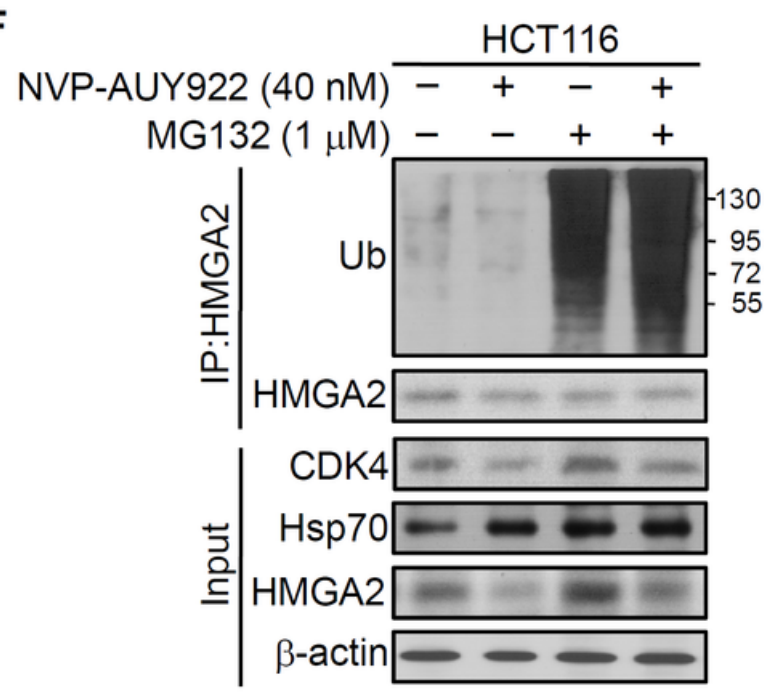

Fig.3 


\section{4}

Expression levels of HMGA2 are responsible for NVP-AUY922 drug sensitivity.

(A) Upper panel: HMGA2 was detected in siHMGA2-transfected HCT116 cells. Bottom panel: HCT116 cells were transfected with control siRNA and siHMGA2 (100 nM) for 48 hours and subsequently incubated with NVP-AUY922 at the indicated concentrations for an additional 48 hours. A cell viability assay was performed to determine the viability of cells treated with various NVP-AUY922 concentrations. Bars, SD $(n=6)$. (B) Western blot analysis of proteins expression of HMGA2, CDK4, and Hsp70 in HCT116 cells transfected with control siRNA or siHMGA2 for 48 hours and subsequently incubated with NVP-AUY922 at the indicated concentrations for an additional 48 hours. (C) Upper panel: Western blotting with anti-GFP antibody of the parental and stable HMGA2-GFP groups of HCT116 cells. Bottom panel: Cell proliferation assays of the parental and stable HMGA2-GFP groups of HCT116 cells treated with NVP-AUY922 at the indicated concentrations for 48 hours. Bars, SD $(n=6) . * p<0.05$, ${ }^{* *} p<0.01,{ }^{* * *} p<0.001$. (D) Western blot analysis of proteins expression of HMGA2, CDK4, and Hsp70 in NVP-AUY922-treated of parental or HMGA2-GFP stable expression of DLD1 cells. All experiments were performed in three independent experiments. 
A
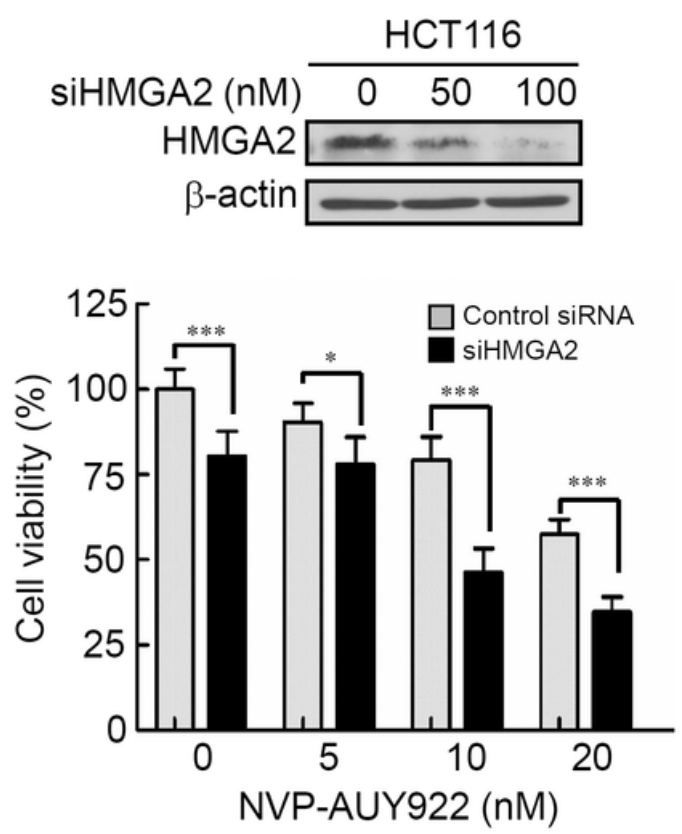

B

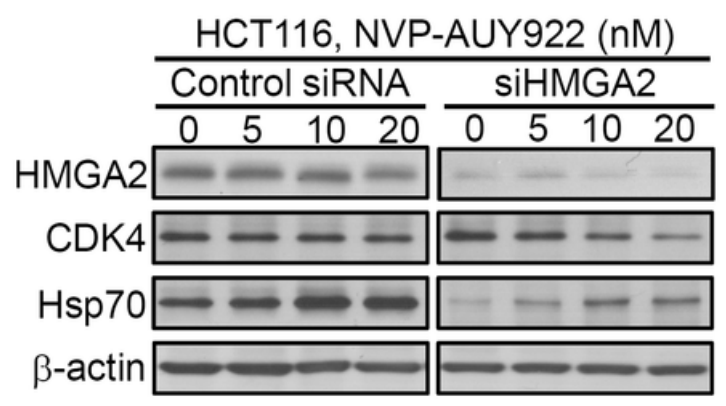

C
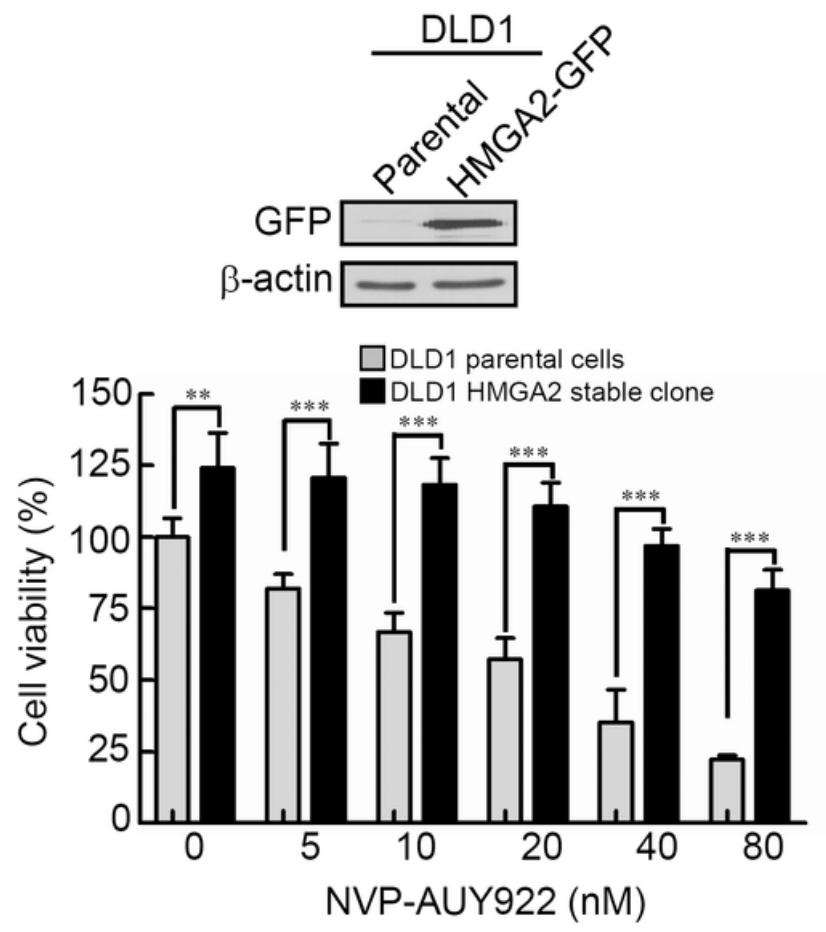

D

\section{Fig.4}




\section{5}

Knockdown HMGA2 expression can enhance the effect of NVP-AUY922-mediated suppression of EMT and migratory ability of HCT116 cells.

(A) siRNA-mediated knockdown of HMGA2 inhibited HMGA2-regulated EMT in HCT116 cells, as revealed by loss of mesenchymal markers Twist, Snail, Slug, Vimentin, and reduction the phosphorylation level of FAK and gain of epithelial marker E-cadherin. (B) Effect of siRNAmediated knockdown of HMGA2 on NVP-AUY922-mediated reversal of mesenchymal character in HCT116 cells. (C and D) Concentration-dependent effects of NVP-AUY922 on the migratory activity of HCT116 cells after 24 hours of treatment. ${ }^{* *} p<0.01, * * *<<0.001$. All experiments were performed in three independent experiments. 
A

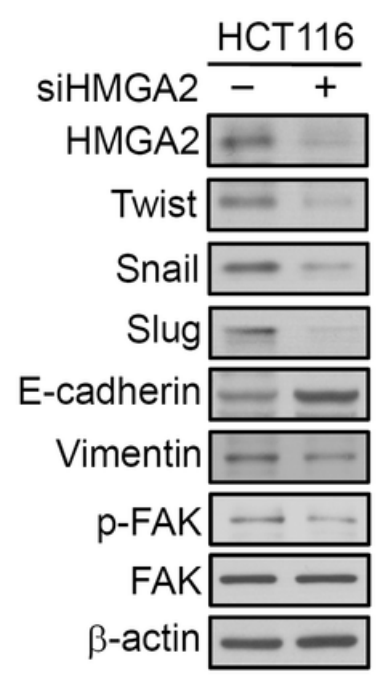

B

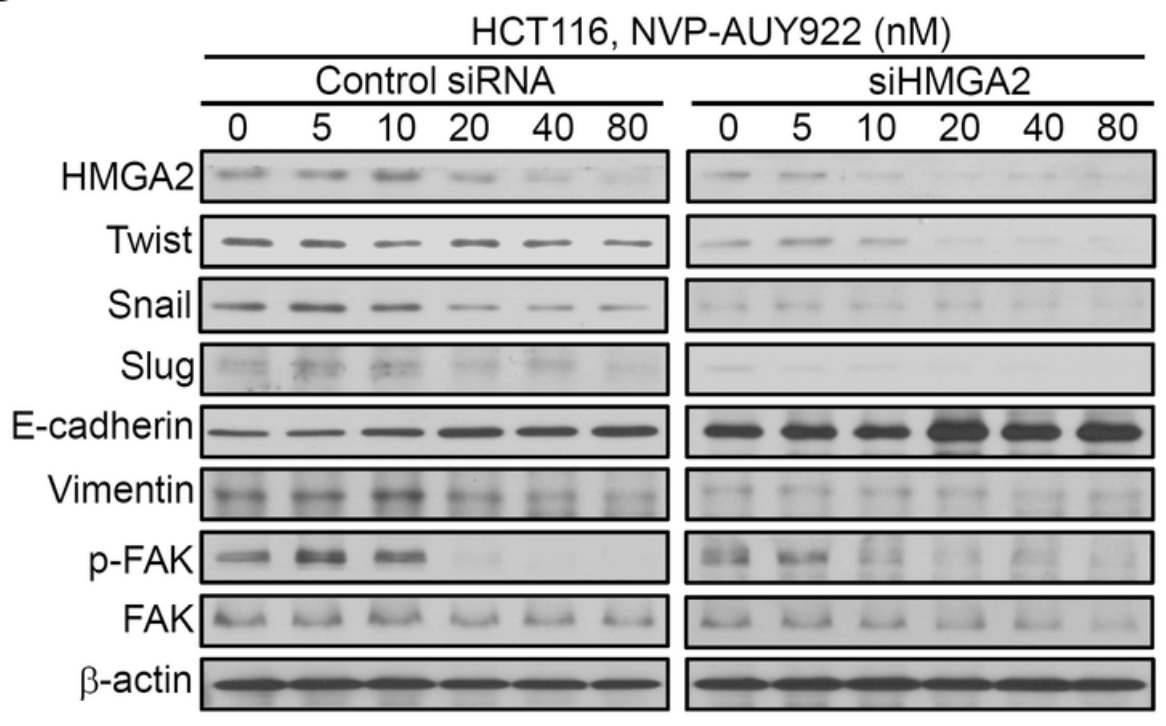

C

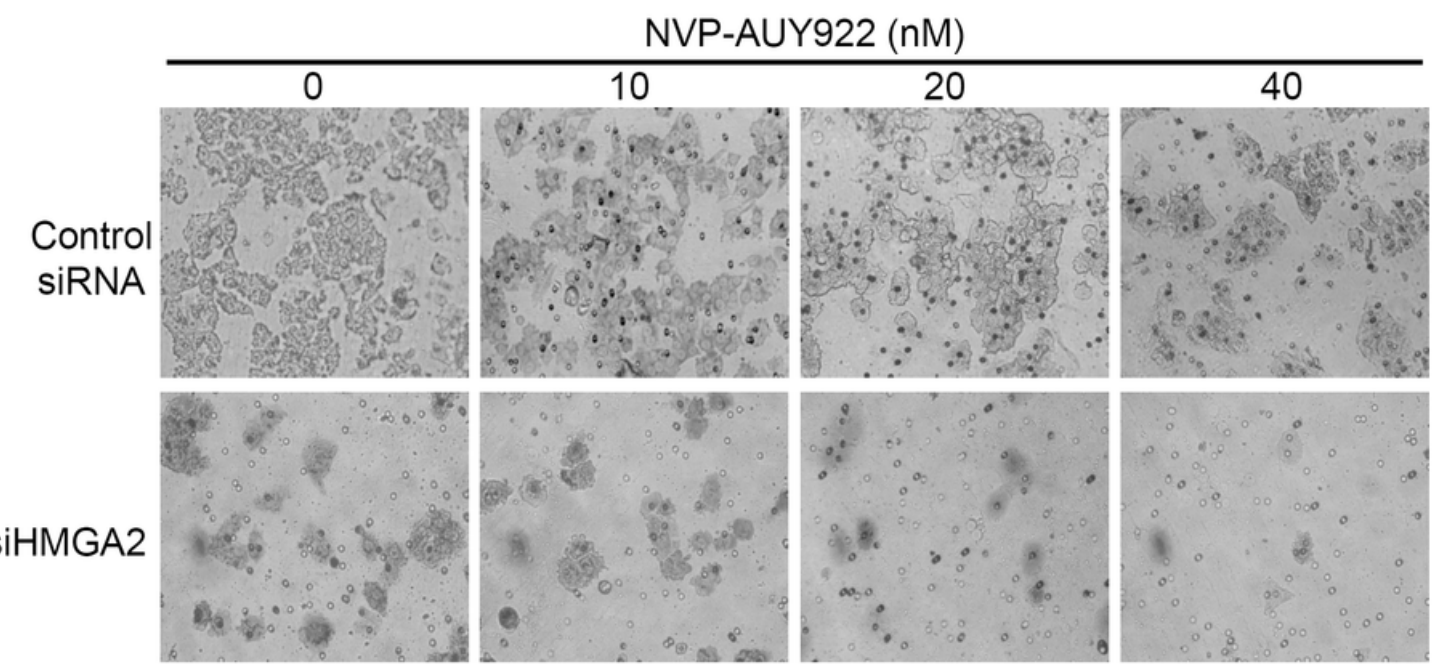

D

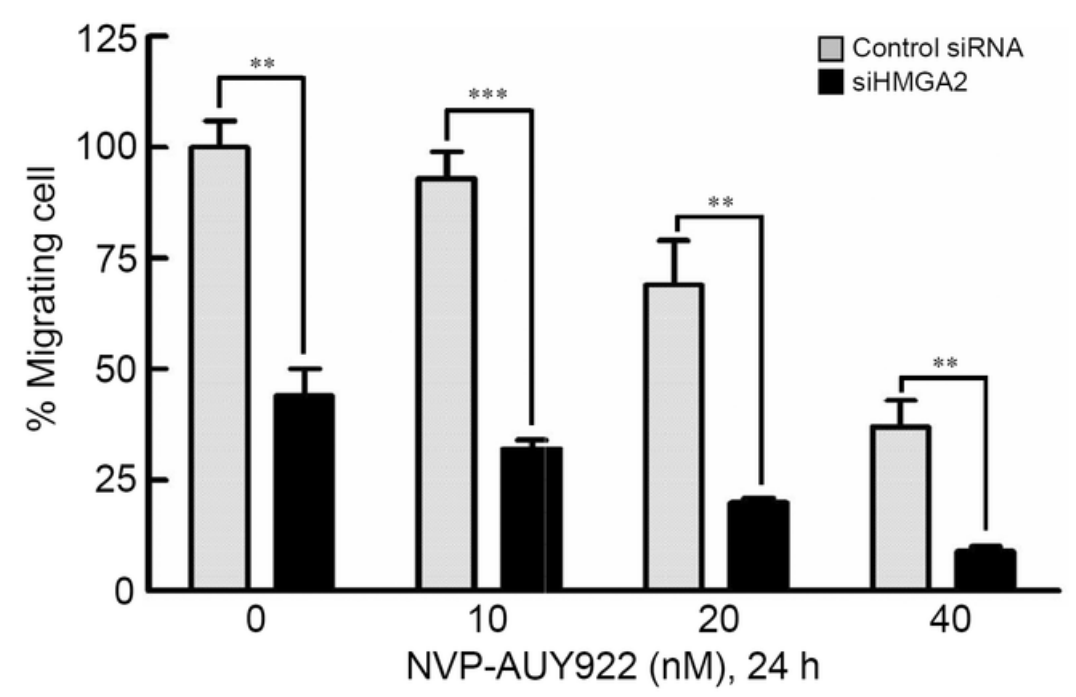

Fig. 5 
6

Human phospho-kinase array analysis in response to NVP-AUY922 treatment in HCT116 cells.

(A) HMGA2-associated kinases, ERK, CREB, and FAK, were significantly downregulated on NVP-AUY922 treatment. (B) Western blotting results of the concentration-dependent effects of NVP-AUY922 on the phosphorylation and expression of ERK and CREB in HCT116 cells. (C) Western blotting revealed the dose effect of AZD6244 on the phosphorylation status of ERK and CREB as well as HMGA2 proteins expression of HCT116 cells. (D) Western blotting results of the concentration-dependent effects of NVP-AUY922 on the phosphorylation and expression of FAK and various EMT effectors of HCT116 cells. All experiments were performed in three independent experiments. 
A
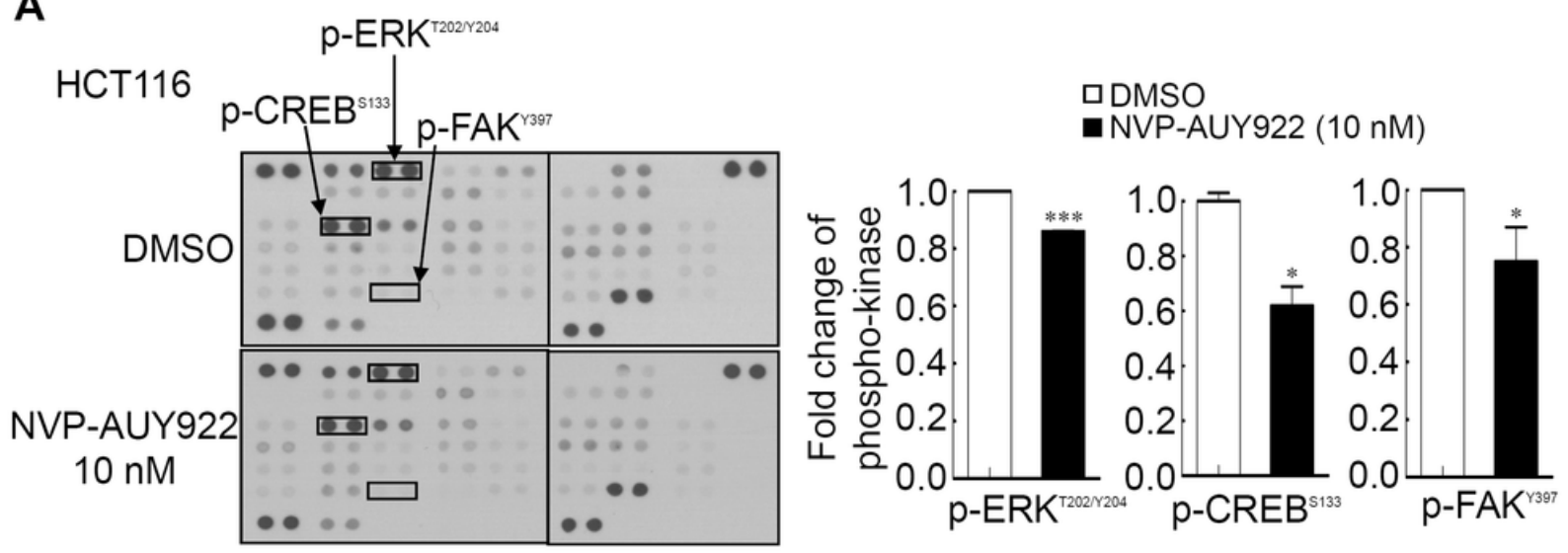

B

C

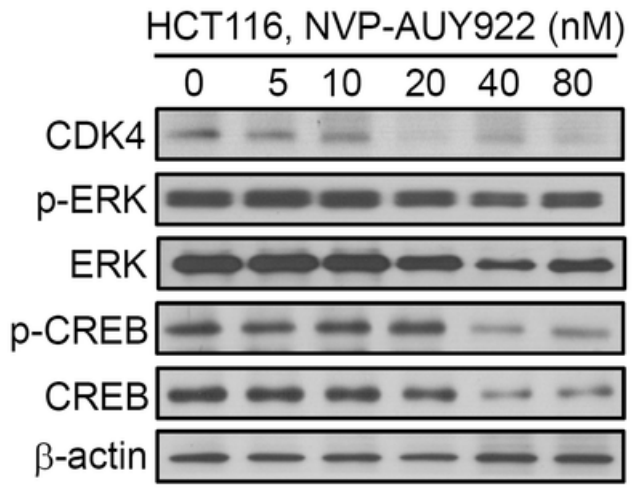

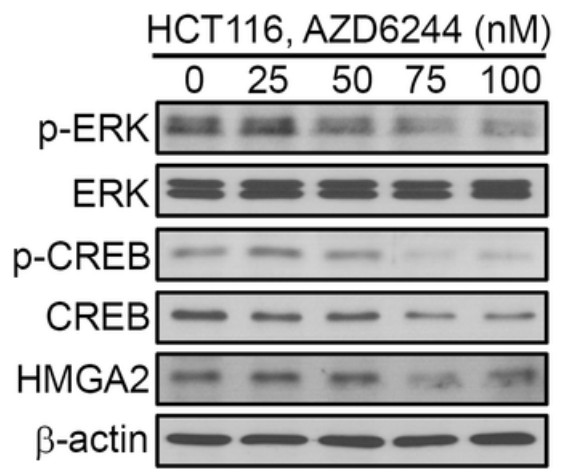

D

HCT116, NVP-AUY922 (nM)

$\begin{array}{llllll}0 & 5 & 10 & 20 & 40 & 80\end{array}$

p-FAK

FAK

$----$

E-cadherin $\longrightarrow-\infty-\infty$

Vimentin

Twist

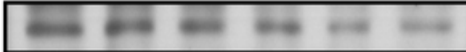

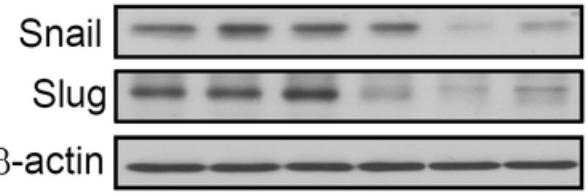

Fig.6 